ARTICLE

DOI: $10.1038 /$ s41467-018-06753-6

\title{
Structural insights on TRPV5 gating by endogenous modulators
}

Taylor E.T. Hughes (10 ${ }^{1}$, Ruth A. Pumroy (1) ${ }^{1}$, Aysenur Torun Yazici (i) ${ }^{2}$, Marina A. Kasimova ${ }^{3}$, Edwin C. Fluck (D) ${ }^{1}$, Kevin W. Huynh4 ${ }^{4}$, Amrita Samanta1, Sudheer K. Molugu', Z. Hong Zhou ${ }^{4}$, Vincenzo Carnevale (i) ${ }^{3}$, Tibor Rohacs ${ }^{2} \&$ Vera Y. Moiseenkova-Bell (1) ${ }^{1}$

TRPV5 is a transient receptor potential channel involved in calcium reabsorption. Here we investigate the interaction of two endogenous modulators with TRPV5. Both phosphatidylinositol 4,5-bisphosphate ( $\left.\mathrm{PI}(4,5) \mathrm{P}_{2}\right)$ and calmodulin $(\mathrm{CaM})$ have been shown to directly bind to TRPV5 and activate or inactivate the channel, respectively. Using cryo-electron microscopy (cryo-EM), we determined TRPV5 structures in the presence of dioctanoyl PI(4,5) $\mathrm{P}_{2}$ and $\mathrm{CaM}$. The $\mathrm{PI}(4,5) \mathrm{P}_{2}$ structure reveals a binding site between the N-linker, S4-S5 linker and $\mathrm{S} 6$ helix of TRPV5. These interactions with $\mathrm{PI}(4,5) \mathrm{P}_{2}$ induce conformational rearrangements in the lower gate, opening the channel. The CaM structure reveals two TRPV5 $\mathrm{C}$-terminal peptides anchoring a single $\mathrm{CaM}$ molecule and that calcium inhibition is mediated through a cation- $\pi$ interaction between Lys116 on the C-lobe of calcium-activated CaM and Trp583 at the intracellular gate of TRPV5. Overall, this investigation provides insight into the endogenous modulation of TRPV5, which has the potential to guide drug discovery.

\footnotetext{
${ }^{1}$ Department of Systems Pharmacology and Translational Therapeutics, Perelman School of Medicine, University of Pennsylvania, Philadelphia, PA 19104, USA. ${ }^{2}$ Department of Pharmacology, Physiology and Neuroscience, New Jersey Medical School, Rutgers University, Newark, NJ 07103, USA. ${ }^{3}$ Institute for Computational Molecular Science, Temple University, Philadelphia, PA 19122, USA. ${ }^{4}$ California NanoSystems Institute, University of California, Los Angeles, CA 90095, USA. These authors contributed equally: Taylor E.T. Hughes, Ruth A. Pumroy, Aysenur Torun Yazici. Correspondence and requests for materials should be addressed to V.Y.M-B. (email: vmb@pennmedicine.upenn.edu)
} 
T he calcium ion is vital for an array of cellular functions, and in the human body the kidneys regulate calcium homeostasis by filtration and reabsorption ${ }^{1}$. Around $99 \%$ of calcium is reabsorbed by the kidney tubules ${ }^{1}$, with $\sim 15 \%$ of that reabsorption occurring via transient receptor potential vanilloid 5 (TRPV5) channels in the distal convoluted tubule and collecting tubule. TRPV5 is specialized for this task and is only expressed in the apical membrane of kidney epithelial cells in the distal convoluted tubule and collecting tubule ${ }^{2,3}$. When open, TRPV5 allows calcium in the urine to flow along a concentration gradient through the channel pore into the cell. This gradient is maintained by the calcium sequestering protein calbindin, which delivers calcium to active transport proteins at the basolateral membrane of the epithelium, which then export the ions into the blood stream ${ }^{2,3}$. Though this mechanism of calcium reabsorption is responsible for only $\sim 15 \%$ of total calcium reabsorbed in the kidney, TRPV5 activity is critical for the homeostatic balance of calcium $^{2,3}$. This is exemplified by TRPV 5 knock out mice, which have been reported to have systemic calcium imbalance in the form of hypercalciuria and bone mineral $\operatorname{loss}^{2,3}$. In humans, single-nucleotide polymorphisms in TRPV5 in AfricanAmerican populations result in a significant increase in calcium reabsorption that is correlated with a lower risk of nephrolithiasis ${ }^{2,3}$. Together, these observations suggest that TRPV5 could be a potential drug target for human disorders involving altered calcium homeostasis.

TRPV5 is a calcium selective channel that displays constitutive activity in the presence of basal levels of the membrane phospholipid phosphatidylinositol 4,5-bisphosphate $\left(\mathrm{PI}(4,5) \mathrm{P}_{2}\right)^{2}$. The TRPV5 channel is tetrameric and consists of classic TRPV family features, including six transmembrane helices (S1-S6), N-terminal ankyrin repeats (ARD), and the TRP domain ${ }^{4}$ (Fig. 1a). TRPV6 is a closely related epithelial $\mathrm{Ca}^{2+}$ channel that shows a high level of sequence homology with TRPV5 ${ }^{3}$; these two channels have a much lower level of sequence homology with other members of the TRPV subfamily. The regulation and biophysical properties of TRPV5 and TRPV6 are similar, and they are functionally quite different from the rest of the TRPV subfamily ${ }^{3}$. PI $(4,5) \mathrm{P}_{2}$ is found in the inner leaflet of the plasma membrane and it has been shown to be essential for the activity of both TRPV5 $5^{5,6}$ and TRPV6 $6^{7}$. For TRPV6, the activating effect of $\mathrm{PI}(4,5) \mathrm{P}_{2}$ was demonstrated in planar lipid bilayers, providing definitive evidence for direct effect on the channel ${ }^{8}$. However, the molecular details of the $\mathrm{PI}(4,5) \mathrm{P}_{2}$ interaction with TRPV5 and TRPV6 are essentially unknown.

To prevent excess flow of calcium ions into the cell, both TRPV5 and TRPV6 have been shown to rapidly inactivate through a calcium-dependent mechanism ${ }^{9}$. Calmodulin (CaM), a calcium sensing protein, has been shown to be involved in this inactivation by directly interacting with the last 30 amino acids of the TRPV5 C-terminus ${ }^{10}$. While the role of the distal C-terminal binding site is well established in CaM-mediated inactivation of TRPV $5^{10}$ and the closely related TRPV $6^{11-13}$, the binding stoichiometry and the conformational changes that take place as a consequence of the binding of $\mathrm{CaM}$ to the channel have been unclear ${ }^{3}$. The absence of this information prevents us from further understanding TRPV5 function and regulation in the kidney.

In this study, we used cryo-electron microscopy (cryo-EM) to uncover the molecular mechanisms of TRPV5 gating via endogenous modulators. Investigating TRPV5 modulation with cryoEM permitted us to gain insight regarding TRPV5 gating and potentially form the basis for rational drug design for the treatment and prevention of hypercalciuria and nephrolithiasis in future studies.

\section{Results}

Structures of lipid-bound and $\mathrm{PI}(4,5) \mathrm{P}_{2}$-bound TRPV5. TRPV5 is part of the tightly regulated system of calcium homeostasis in the human body ${ }^{2}$. Two endogenous mechanisms of regulation, binding of $\mathrm{PI}(4,5) \mathrm{P}_{2}$ or $\mathrm{CaM}$ to TRPV5, result in activation or inactivation of the channel ${ }^{13}$, respectively. To understand these mechanisms of modulation, first we used cryoEM to determine the structure of detergent solubilized full-length rabbit TRPV 5 in the presence of the $200 \mu \mathrm{M}$ dioctanoyl $\left(\mathrm{diC}_{8}\right) \mathrm{PI}$ $(4,5) \mathrm{P}_{2}$, a soluble form of $\mathrm{PI}(4,5) \mathrm{P}_{2}$. This concentration is $\sim$ three times the $\mathrm{EC}_{50}$ of $\mathrm{diC}_{8} \mathrm{PI}(4,5) \mathrm{P}_{2}$ for TRPV6, and TRPV5 has a slightly higher apparent affinity for $\mathrm{diC}_{8} \mathrm{PI}(4,5) \mathrm{P}_{2}{ }^{14}$, thus this concentration was expected to result in near saturation of TRPV5 with $\operatorname{diC}_{8} \mathrm{PI}(4,5) \mathrm{P}_{2}$. During the cryo-EM structure determination process, one stable class emerged and was refined to an overall resolution of $3.9 \AA$ (Fig. 1b, Supplementary Figure 1, Supplementary Figure 2, Supplementary Table 1). The vast majority of this TRPV 5 cryo-EM map is at high resolution $(3-3.5 \AA)$ as side chains are clearly visible in the transmembrane region of the channel (Supplementary Figure 1, Supplementary Figure 3). This allowed for accurate model building in both the transmembrane domain (TMD) and the ankyrin repeat domain (ARD) (Fig. 1c, Supplementary Figure 3). Highly flexible areas such as the very distal C-terminal and $\mathrm{N}$-terminal were unable to be resolved. Interestingly, five non-protein densities per monomer were identified in this cryo-EM map (Fig. 1b). None of these could be identified as $\mathrm{diC}_{8} \mathrm{PI}(4,5) \mathrm{P}_{2}$, and were attributed to annular lipids that had high enough affinity for TRPV5 to be co-purified with the protein. Similarly shaped non-protein densities were also assigned as lipids in a recently reported human TRPV6 cryo-EM structure $^{15}$. Therefore, we will refer to this channel reconstruction as lipid-bound TRPV5.

The lipid-bound TRPV5 pore contains three residues that are involved in pore constriction: Asp542, Ile575, and Trp583 (Fig. 1d). Unlike the previously published structure of TRPV5 ${ }^{4}$, there is clear density in the lipid-bound TRPV5 map indicating that the four Asp542 that constitute the highly specific $\mathrm{Ca}^{2+}$ selectivity filter are pointing directly into the pore in a similar fashion to the rat TRPV6 crystal structures ${ }^{16,17}$. The lipid-bound TRPV5 lower gate appears to consist of Ile575 and Trp583 (Fig. 1d, e). These residues do not constrict the pore to the point of blocking ion translocation, indicating that the lower gate is open (Fig. 1e), as seen in the human TRPV6 cryo-EM structure ${ }^{15}$. This suggests that the pore of the lipid-bound TRPV5 structure may be in a closed pre-open conformation.

Based on these findings, we decided to increase $\mathrm{diC}_{8} \mathrm{PI}(4,5) \mathrm{P}_{2}$ concentration to $400 \mu \mathrm{M}$ and use nanodiscs to capture TRPV5 in the $\mathrm{PI}(4,5) \mathrm{P}_{2}$-bound state. During the cryo-EM structure determination process we noted that even before masking and $3 \mathrm{D}$ classification in RELION $^{18,19}$ the initial $3 \mathrm{D}$ reconstruction contained a non-protein density that resembled $\mathrm{diC}_{8} \mathrm{PI}(4,5) \mathrm{P}_{2}$ in both size and shape (Fig. 2a, Supplementary Figure 4). Next, we used particle subtraction and $3 \mathrm{D}$ classification that was focused on the $\mathrm{diC}_{8} \mathrm{PI}(4,5) \mathrm{P}_{2}$-binding site using a mask which included the entire TRP domain helix, the base of the S6 helix, and the head group of the $\mathrm{diC}_{8} \mathrm{PI}(4,5) \mathrm{P}_{2}$ (Supplementary Figure 4). This classification yielded three classes with good features: one without $\mathrm{diC}_{8} \mathrm{PI}(4,5) \mathrm{P}_{2}$ and two with $\mathrm{diC}_{8} \mathrm{PI}(4,5) \mathrm{P}_{2}$ (Supplementary Figure 4). The class without $\operatorname{diC}_{8} \mathrm{PI}(4,5) \mathrm{P}_{2}$ yielded a TRPV5 structure at $4.4 \AA$, which clearly resembled the lipid-bound TRPV5 in detergent (Supplementary Figure 5). Due to high similarity between the detergent and nanodisc lipid-bound TRPV5 structures (Supplementary Figure 5), we will only describe the lipid-bound TRPV5 in detergent as it was determined at a higher resolution (Supplementary Figure 1, Supplementary Figure 3, Supplementary Figure 5). The two classes with $\mathrm{diC}_{8} \mathrm{PI}(4,5) \mathrm{P}_{2}$ density, after 

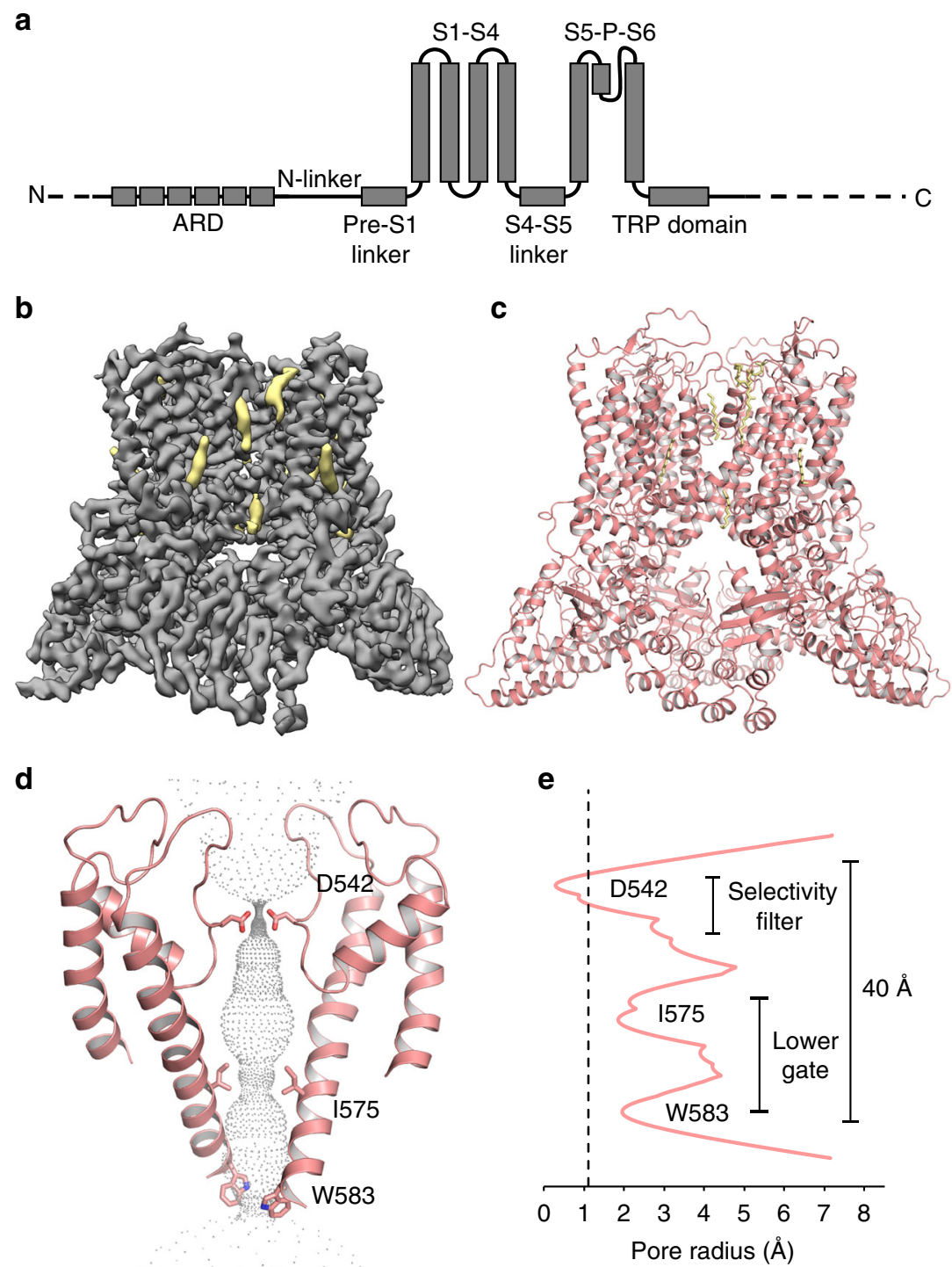

Fig. 1 Lipid-bound TRPV5 structure in detergent. a A schematic representation of the TRPV5 domains per channel monomer. Dashed lines indicate regions for which a model could not be built. b Density map of lipid-bound TRPV5 at $3.9 \AA$ resolution. Density for TRPV 5 is shown in gray and the densities attributed to annular lipids are shown in khaki. c Cartoon representation of the lipid-bound TRPV 5 model. The TRPV 5 tetramer is depicted as pink cartoons and annular lipids as khaki sticks. $\mathbf{d}$ Cartoon representation of the lipid-bound TRPV5 pore highlighting the three constriction points in the selectivity filter and lower gate. e Plot of pore radii of lipid-bound TRPV5 as a function of distance through the pore. The dotted line indicates the radius of a dehydrated calcium ion

additional classification and refinement, produced a single TRPV5 structure in a diC 8 PI $(4,5) \mathrm{P}_{2}$-bound state at $4.0 \AA$ (Fig. $2 \mathrm{~b}$, Supplementary Figure 6, Supplementary Figure 7, Supplementary Table 1). All structures identified in nanodiscs revealed non-protein densities in similar positions to those attributed to annular lipids in the lipid-bound TRPV5 structure in detergent (Fig. 1b, Fig. 2a, b, Supplementary Figure 5).

The $\mathrm{PI}(4,5) \mathrm{P}_{2}$-binding site in the TRPV5 channel is located between the N-linker (Arg302, Arg305), S4-S5 linker (Lys484), and the S6 helix (Arg584) of the channel (Fig. 2c). These results show an excellent correlation with predictions by earlier molecular dynamics (MD) simulations performed on the homologous TRPV6 channel (Fig. 2d, Supplementary Figure 8). These simulations predicted that residues Arg302, Arg305, and Lys484 are involved in the TRPV6 interaction with $\mathrm{PI}(4,5) \mathrm{P}_{2}$ (Fig. 2d, Supplementary Figure 8). Moreover, when these predicted PI $(4,5) \mathrm{P}_{2}$-interacting TRPV6 residues (Arg302,
Arg305, Lys484) were mutated to glutamine, the channel had increased sensitivity to depletion of $\mathrm{PI}(4,5) \mathrm{P}_{2}$ with high concentrations of wortmannin, which inhibits PI4-kinases (Fig. 2e, Supplementary Figure 9). This result indicates reduced apparent affinity for $\mathrm{PI}(4,5) \mathrm{P}_{2}$, which is expected if a $\mathrm{PI}(4,5) \mathrm{P}_{2^{-}}$ interacting residue is mutated ${ }^{20}$. The current amplitudes of the Arg302Gln and Lys484Gln mutants were also significantly lower than wild type TRPV6 (Supplementary Figure 9), which is consistent with decreased apparent affinity of the channel for PI $(4,5) \mathrm{P}_{2}$. The Arg305 residue showed a less clear contact with PI $(4,5) \mathrm{P}_{2}$ compared to Arg302 and Lys484, interacting with only three out of four subunits in MD simulations (Supplementary Figure 8). Accordingly, this mutant showed a less pronounced trend to be inhibited more by wortmannin and had a smaller amplitude, but these effects did not reach statistical significance from wild type (Fig. 2e, Supplementary Figure 9). When we mutated residues Arg302 and Lys484 to glutamine in TRPV5 and 
a

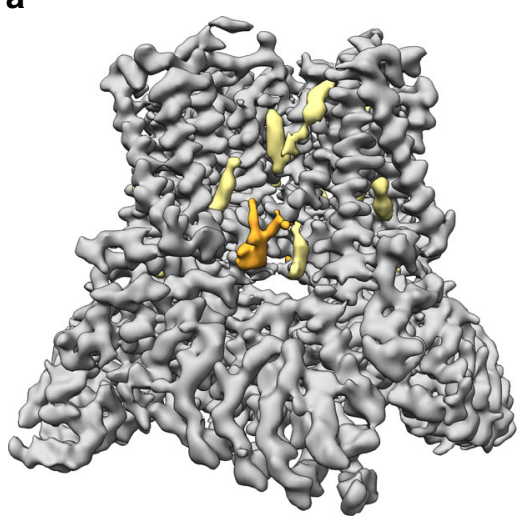

d

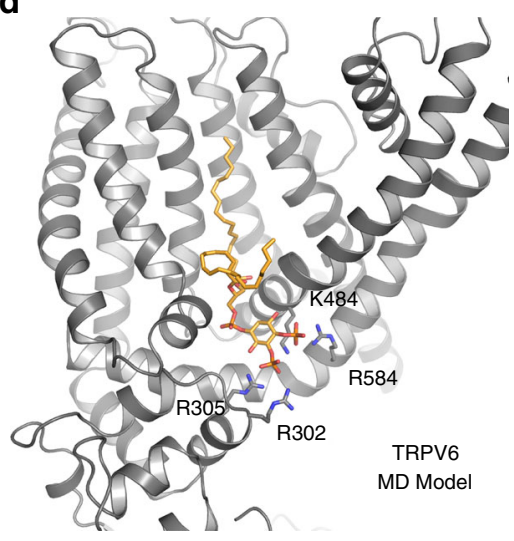

b

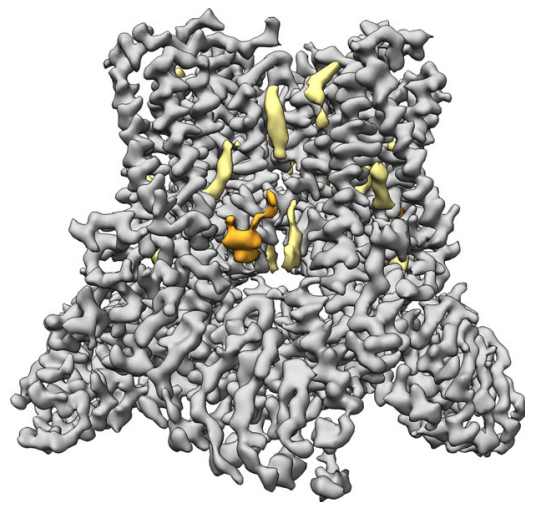

e

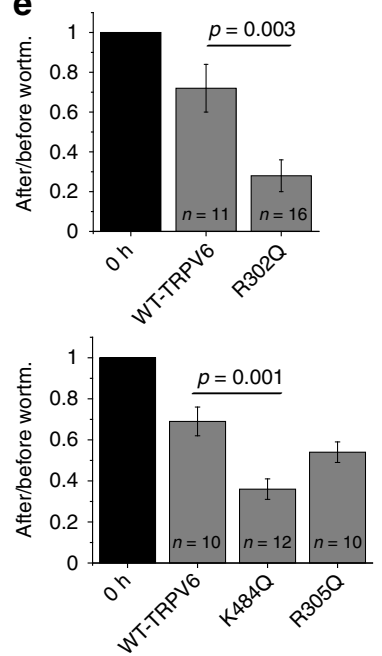

c

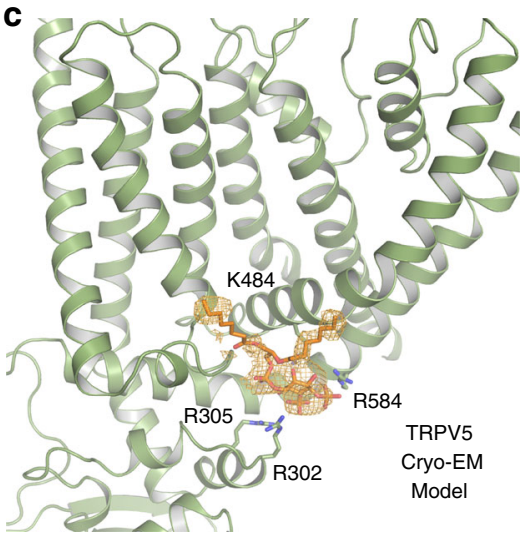

f

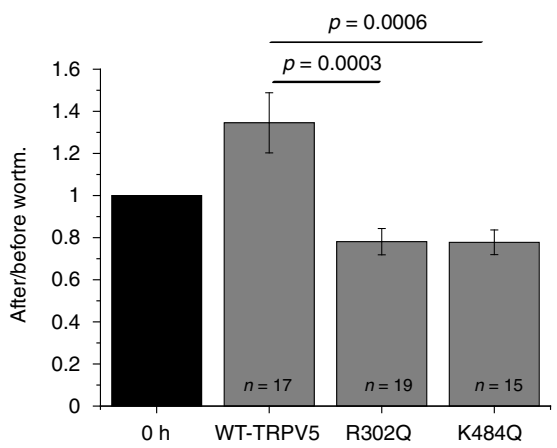

Fig. $2 \mathrm{PI}(4,5) \mathrm{P}_{2}$-bound TRPV5 structure and $\mathrm{PI}(4,5) \mathrm{P}_{2}$-bound TRPV6 modeling. a The initial reconstruction of $\mathrm{PI}(4,5) \mathrm{P}_{2}$-bound TRPV5 in nanodiscs before masking and 3D classification. TRPV5 density is shown in gray, annular lipids are shown in khaki, and $\mathrm{PI}(4,5) \mathrm{P}_{2}$ is shown in orange. $\mathbf{b} \mathrm{PI}(4,5) \mathrm{P}_{2}$-bound TRPV5 cryo-EM density in nanodiscs after focused 3D classification. TRPV5 density is shown in gray, annular lipids are shown in khaki, and PI(4,5) $\mathrm{P}_{2}$ is shown in orange. c Zoomed-in view of the TRPV5 $\mathrm{PI}(4,5) \mathrm{P}_{2}$-binding pocket. The $\mathrm{PI}(4,5) \mathrm{P}_{2}$-binding site in the TRPV5 channel is located between the $\mathrm{N}$-linker (R302, R305), S4-S5 linker (K484), and the S6 helix (R584) of the channel. d A model produced by molecular dynamics (MD) simulations of the predicted interaction between the homologous TRPV6 channel and $\mathrm{PI}(4,5) \mathrm{P}_{2}$. e Neutralization of key $\mathrm{PI}(4,5) \mathrm{P}_{2}$ interacting residues increases sensitivity to depletion of $\mathrm{PI}(4,5) \mathrm{P}_{2}$ in TRPV6. The TRPV6 currents in oocytes measured before and after incubation with $35 \mu \mathrm{M}$ wortmannin for $1 \mathrm{~h}$. Current values after wortmannin treatment are normalized to current values before treatment. $P$-values for significance values are shown after rounding to the first non-zero digit (analysis of variance). f Neutralization of key PI(4,5) $\mathrm{P}_{2}$ interacting residues increases sensitivity to depletion of PI(4,5) $\mathrm{P}_{2}$ in TRPV5. The TRPV5 currents in oocytes measured before and after incubation with $35 \mu \mathrm{M}$ wortmannin for $1 \mathrm{~h}$. Current values after wortmannin treatment are normalized to current values before treatment. $P$-values for significance values are shown after rounding to the first non-zero digit (analysis of variance). The sample size $(n)$ indicates the number of individual oocytes tested, from at least two different oocyte preparations. Error bars represent \pm SEM

performed analogous experiments, we observed that the TRPV5 channel also had significantly increased sensitivity to the depletion of $\mathrm{PI}(4,5) \mathrm{P}_{2}$ with high concentrations of wortmannin, suggesting that both TRPV5 and TRPV6 share a similar PI(4,5) $\mathrm{P}_{2}$-binding site (Fig. 2f). Unlike for TRPV6, current amplitudes for these mutants were not statistically significantly different from wild type TRPV5 (Supplementary Figure 9).

Based on the structure of $\mathrm{PI}(4,5) \mathrm{P}_{2}$-bound TRPV5 compared to the lipid-bound TRPV5, the binding of $\mathrm{diC}_{8} \mathrm{PI}(4,5) \mathrm{P}_{2}$ to TRPV5 appears to induce conformational changes related to channel activity. The pore of the channel widened, which could allow for the flow of hydrated $\mathrm{Ca}^{2+}$ ions (Fig.3a-d). Specifically, the Asp542 residues, which line the selectivity filter of the channel and coordinate $\mathrm{Ca}^{2+}$ ions, point upwards in the extracellular vestibule of the channel instead of towards the ion conduction pathway as seen in the lipid-bound structure (Fig. 3d). The distance between the center of the carboxylate oxygen atoms of the Asp542 residues in this structure is $\sim 3 \AA$, which would allow for partially hydrated $\mathrm{Ca}^{2+}$ ion to flow though the pore of the channel (Fig. 3c, d). The lower gate of the channel is also wide open $(\sim 12 \AA$ compared to $\sim 8 \AA$ in the lipid-bound state) due to the movement of the $\mathrm{S} 6$ helix and Trp583 upon binding of $\mathrm{diC}_{8}$ $\mathrm{PI}(4,5) \mathrm{P}_{2}$ (Fig. 3e), which would facilitate the movement of partially hydrated $\mathrm{Ca}^{2+}$ ions through the pore (Fig. 3C). Additionally, $\mathrm{diC}_{8} \mathrm{PI}(4,5) \mathrm{P}_{2}$ binding caused global conformational changes in the S6 and TRP helices, as well as the S4-S5 linker when compared to the lipid-bound structure (Fig. 3f).

Comparison between the $\mathrm{diC}_{8} \mathrm{PI}(4,5) \mathrm{P}_{2}$-bound and lipidbound TRPV5 structures revealed details of the conformational changes induced by $\mathrm{PI}(4,5) \mathrm{P}_{2}$ binding (Fig. 4). The inositol ring of the $\mathrm{diC}_{8} \mathrm{PI}(4,5) \mathrm{P}_{2}$ head group is positioned close to the lower gate of the channel, where phosphate groups at position four and five can interact directly with Arg584 and Arg302, respectively (Fig. 4a, c). Specifically, the phosphate group at the position four 
a
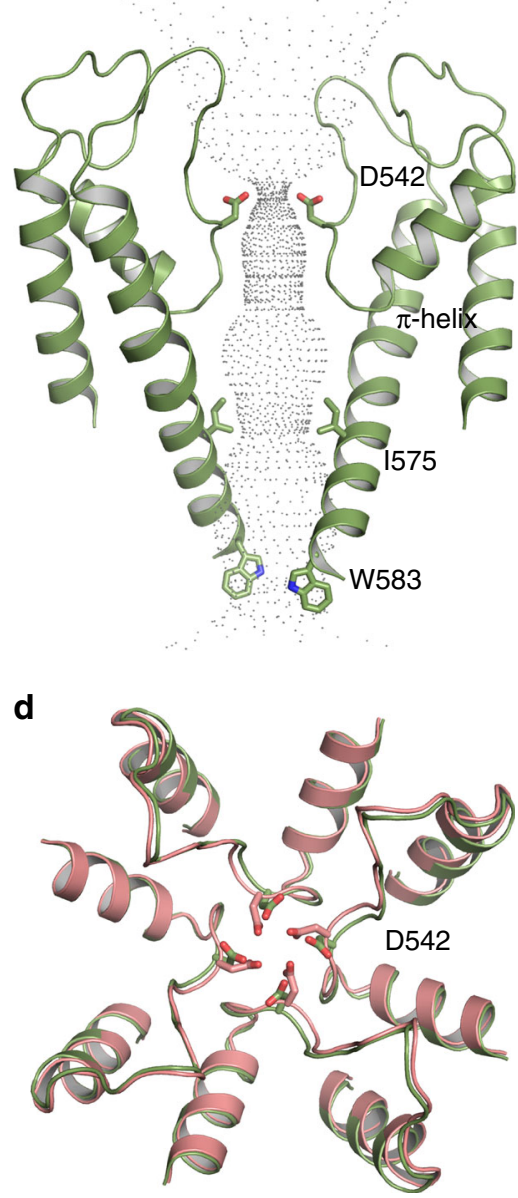

b

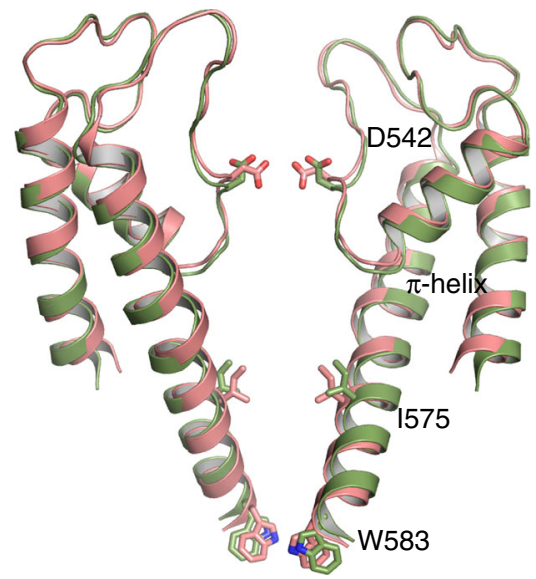

e

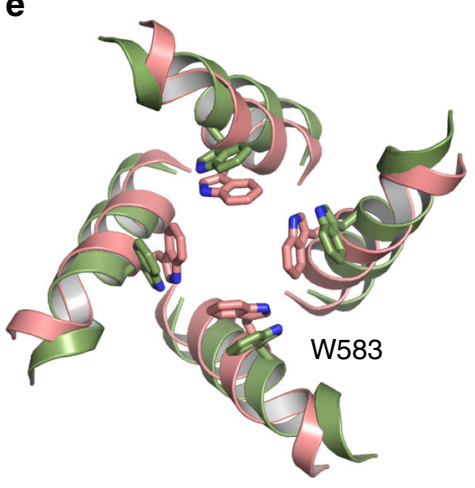

C
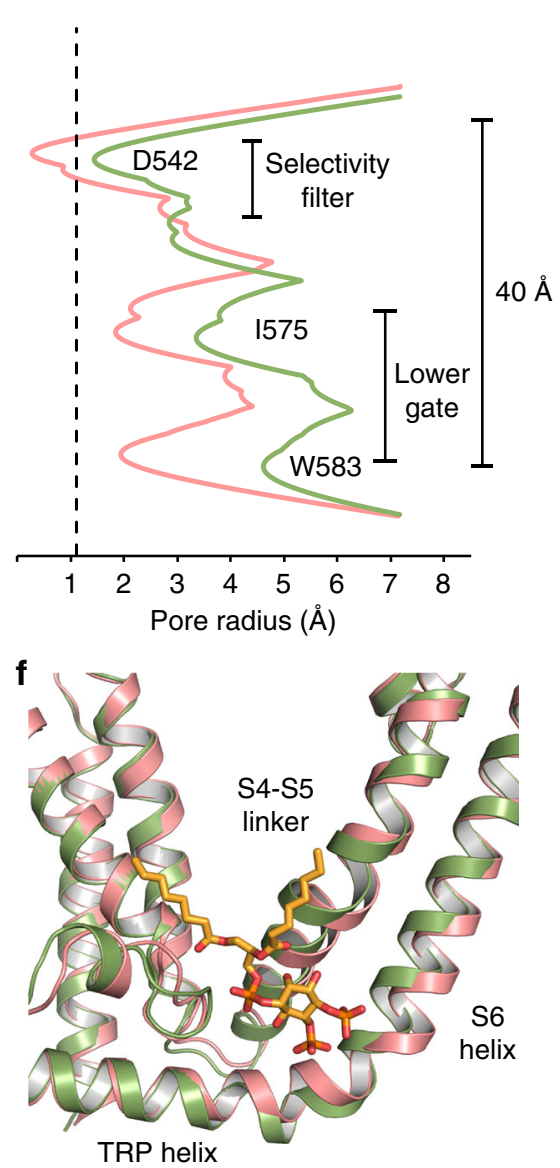

Fig. 3 Comparison between lipid-bound and PI(4,5) $\mathrm{P}_{2}$-bound TRPV5. a The pore diagram of PI(4,5) $\mathrm{P}_{2}$-bound TRPV5 is shown in green. $\mathbf{b}$ Lipid-bound TRPV5 (pink) superimposed onto the PI $(4,5) \mathrm{P}_{2}$-bound (green) pore diagram shows a slight shift in the $\mathrm{S} 6$ helices and important gating residues originating

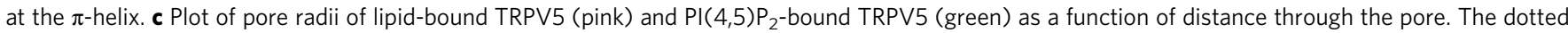
line indicates the radius of a calcium ion. $\mathbf{d}$ Extracellular view of the tetrameric selectivity filter of lipid-bound (pink) and PI(4,5) $\mathrm{P}_{2}$-bound TRPV5 (green). e Intracellular view of the tetrameric lower gate of lipid-bound (pink) and PI(4,5) $\mathrm{P}_{2}$-bound TRPV5 (green). $\mathbf{f}$ Zoomed-in view of the overlayed TMDs of lipid-bound (pink) and $\mathrm{PI}(4,5) \mathrm{P}_{2}$-bound TRPV5 (green). $\mathrm{DiC}_{8} \mathrm{PI}(4,5) \mathrm{P}_{2}$ is shown in orange sticks

forms a salt bridge with the Arg584 on the S6 helix. This interaction is facilitated by the rotation of the Arg584 towards PI $(4,5) \mathrm{P}_{2}$, which pulls the $\mathrm{S} 6$ helix away from the center of the pore, starting at the $\pi$-helix (Fig. $3 b$ ), and causes an outward shift of the S4-S5 linker (Fig. 3f). The rotation of Arg584 also induces the lengthening of the S6 helix and shortens the TRP helix (Fig. 3f, Fig. $4 \mathrm{a}, \mathrm{b})$ : in the lipid-bound TRPV5 structure, the transition between the S6 helix and the TRP helix occurs between Arg584 and Val585, while in the $\mathrm{diC}_{8} \mathrm{PI}(4,5) \mathrm{P}_{2}$-bound TRPV5 structure this transition occurs between residues Gln587 and Glu588. The extension and rotation of the S6 helix pulls Trp583 out of the pore and stabilizes its position through an interaction with Gln587, thus opening the lower gate. The shortening of the TRP helix causes Glu588 to pull away from Arg302, allowing Arg302 to interact with the phosphate group at position five of the $\mathrm{diC}_{8}$ $\mathrm{PI}(4,5) \mathrm{P}_{2}$ head group (Fig. 4c, d). Additionally, Lys484 interacts with the $\mathrm{diC}_{8} \mathrm{PI}(4,5) \mathrm{P}_{2}$ inositol ring, which further electrostatically stabilizes the position of $\mathrm{diC}_{8} \mathrm{PI}(4,5) \mathrm{P}_{2}$ in its binding pocket (Fig. 4c, d, Supplementary Movie 1).

TRPV5 inhibition by calmodulin. In order to investigate the mechanism of TRPV5 inactivation by CaM, we incubated detergent solubilized full-length rabbit TRPV5 with calciumactivated rat CaM in a 1:20 molar ratio in the presence of $10 \mathrm{mM}$ $\mathrm{CaCl}_{2}$. The stoichiometry of TRPV 5 to CaM has been speculated to be between one and four molecules of CaM per tetramer ${ }^{3,6,21-23}$ and the affinity between CaM and the TRPV5 C-terminus estimated at $\sim 0.3 \mu \mathrm{M}^{24}$, therefore the high molar ratio was used to ensure TRPV5 saturation with CaM. This sample yielded a cryoEM map at $4.4 \AA$ without applied symmetry (Fig. 5a, Supplementary Figure 10, Supplementary Figure 11, Supplementary Figure 12, Supplementary Table 1). In this structure, a single CaM molecule is bound to the intracellular section of TRPV5 at the base of the pore (Fig. 5a). Both lobes of CaM are resolved and each bound to different sections of the TRPV5 C-terminus (Fig. 5a, b, Supplementary Figure 12). Despite the interaction with CaM, the CaM-bound conformation of TRPV5 is almost identical to the lipid-bound conformation of TRPV5, with an RMSD of $\sim 0.6 \mathrm{~A}$ overall and a very similar pore profile (Fig. $5 \mathrm{c}, \mathrm{d}$ ). Additionally, annular lipids were also identified in the CaM-bound TRPV5 structure in similar positions to those in the lipid-bound TRPV 5 structure in detergent (Figs. $1 \mathrm{~b}$ and $5 \mathrm{a}$ ).

$\mathrm{CaM}$ is bound with the C-lobe at the base of the pore and the $\mathrm{N}$-lobe resting against the ankyrin repeats of two adjacent TRPV5 monomers (Fig. 5a, b). A portion of the CaM N-lobe 

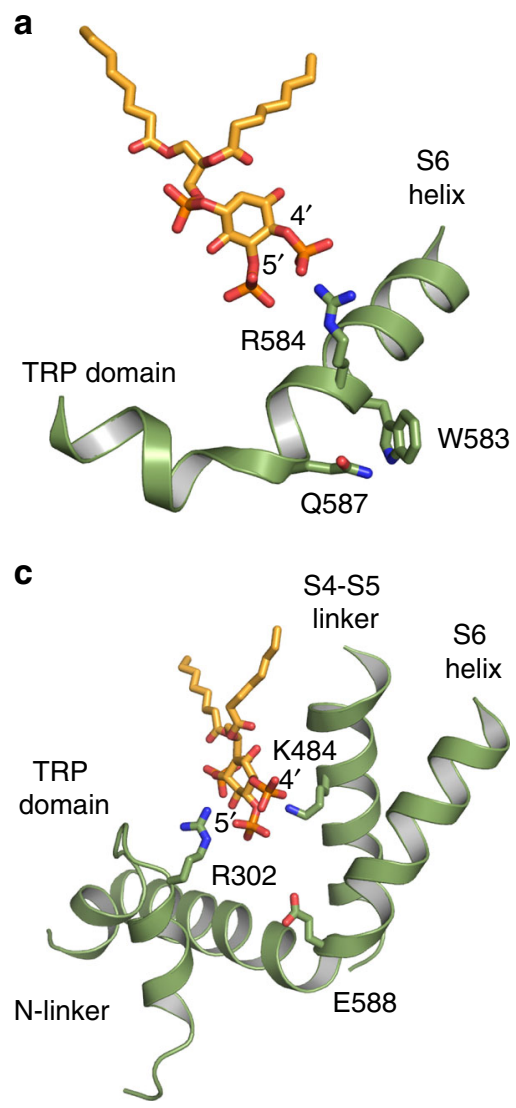

b

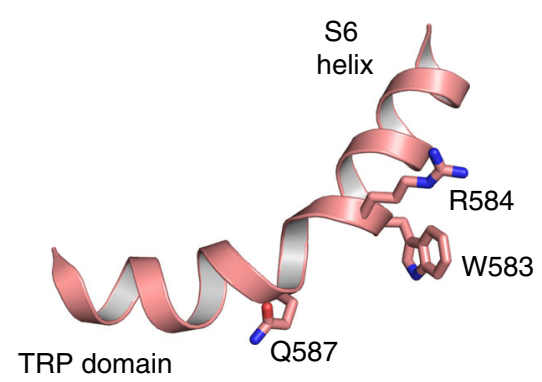

d

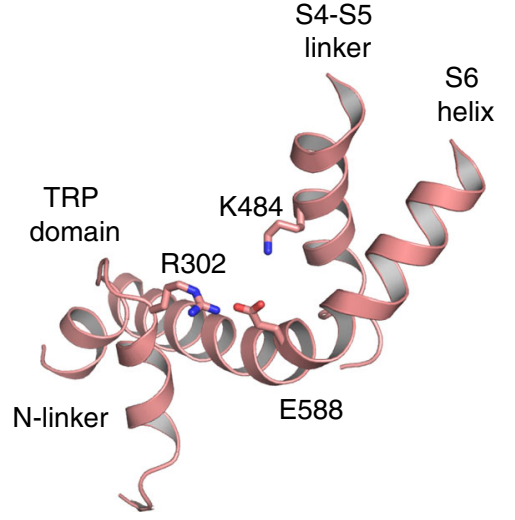

Fig. 4 Interactions of TRPV5 with PI(4,5) $\mathrm{P}_{2}$. a View of the lower $\mathrm{S} 6$ helix and TRP domain of PI(4,5) $\mathrm{P}_{2}$-bound TRPV5. The PI(4,5) $\mathrm{P}_{2}$-bound TRPV5 is shown in green and the PI(4,5) $\mathrm{P}_{2}$ in orange. $\mathbf{b}$ View of the lower $\mathrm{S} 6$ helix and TRP domain of the lipid-bound TRPV5 structure (pink). c The 5' phosphate of PI(4,5) $\mathrm{P}_{2}$ (orange) interacts with R302 of the N-linker and K484 of the S4-S5 linker in the PI(4,5) $\mathrm{P}_{2}$-bound TRPV5 structure. $\mathbf{d}$ The lipid-bound structure showing the N-linker, S4-S5 linker, S6 helix, and TRP domain in pink

(Gly41-Ile64) and the linker between the CaM two lobes (Lys78-Ser82) are visible only as broken density and were excluded from the model (Fig. 6a). A peptide from the C-terminus of TRPV5 (His699-Thr709) is bound to the CaM C-lobe, with TRPV5 Trp702 making critical contacts with the CaM-binding pocket, as previously reported ${ }^{23}$ (Fig. 6a, c). This peptide was arbitrarily assigned to TRPV5 chain A in the model, but no connections could be seen between the density for this peptide and any TRPV5 monomer. An extension of the TRPV5 chain A C-terminus (Asn640-Lys652) forms a helix and is bound to the hydrophobic binding pocket of the CaM N-lobe (Fig. 6b). This interaction is mediated by numerous hydrophobic contacts: Val644, Leu645, Val648, and Phe651 on the TRPV5 side and Phe20, Leu33, Val36, and Leu64 on the CaM side (Fig. 6a, d). A loop of the CaM C-lobe (Gly114-Thr118) crosses over the bottom of the TRPV5 pore, placing Lys116 directly into the pore. The $\varepsilon$-amino group of Lys116 sits in between the four Trp583 residues of the lower gate, forming a cation $-\pi$ interaction and thereby blocking the flow of ions through the pore (Fig. 6e). To test the functional importance of Trp583 in TRPV5 inhibition by CaM, we performed excised inside out patch clamp experiments. Wild type TRPV5 currents were robustly inhibited by $\mathrm{Ca}^{2+}-\mathrm{CaM}$ $\left(0.2 \mu \mathrm{M}\right.$ CaM, $3 \mu \mathrm{M}$ free $\left.\mathrm{Ca}^{2+}\right)$ applied to the inner leaflet of the patch membrane (Fig. 6f, h), as reported previously for TRPV $6^{13}$. On the other hand, the Trp583 to Leu mutant of TRPV5 was essentially not inhibited by $\mathrm{Ca}^{2+}$-CaM (Fig. $6 \mathrm{~g}$, h). Thus, this cation $-\pi$ interaction appears to be critical for CaM-induced inhibition of TRPV5 (Fig. 6f-h).

\section{Discussion}

Here we have presented three structures of TRPV5, one in the presence of endogenous lipids that are likely to be involved in maintaining the structure of TRPV5, one bound to its endogenous activator, $\mathrm{PI}(4,5) \mathrm{P}_{2}$, and one bound to its endogenous inhibitor, CaM. The high-resolution, lipid-bound TRPV5 has allowed for more accurate model building of TRPV5 than available previously ${ }^{4}$. This structure also identified the binding pockets for several high affinity lipids which were consistently seen in all TRPV5 structures presented here. These binding pockets have the potential to be druggable areas of the TRPV5 channel as they are both membrane and solvent accessible and are in contact with regions that have been reported to be able to transmit conformational rearrangements to the pore in other TRPV family channels ${ }^{4,15,25}$.

$\mathrm{PI}(4,5) \mathrm{P}_{2}$ is a conserved positive regulator of most TRP channels ${ }^{20}$. The TRPV5-bound $\mathrm{PI}(4,5) \mathrm{P}_{2}$ structure revealed directly how a TRP channel is opened by this important endogenous lipid at the molecular level. Similar binding sites for PI $(4,5) \mathrm{P}_{2}$ have been theoretically predicted for TRPV ${ }^{26}$ and TRPV6 ${ }^{14}$ channels using computational modeling and experimental approaches, suggesting that the S4-S5 linker plays an essential role in this interaction. Our cryo-EM structure directly demonstrates an interaction between $\mathrm{PI}(4,5) \mathrm{P}_{2}$ and TRPV5 through the N-linker, the S4-S5 linker and the S6 helix of each TRPV monomer (Fig. 2). Comparison between the $\mathrm{PI}(4,5) \mathrm{P}_{2^{-}}$ bound and lipid-bound TRPV5 structures reveals that binding of $\mathrm{PI}(4,5) \mathrm{P}_{2}$ near to the lower gate of the TRPV5 channel induces 
a
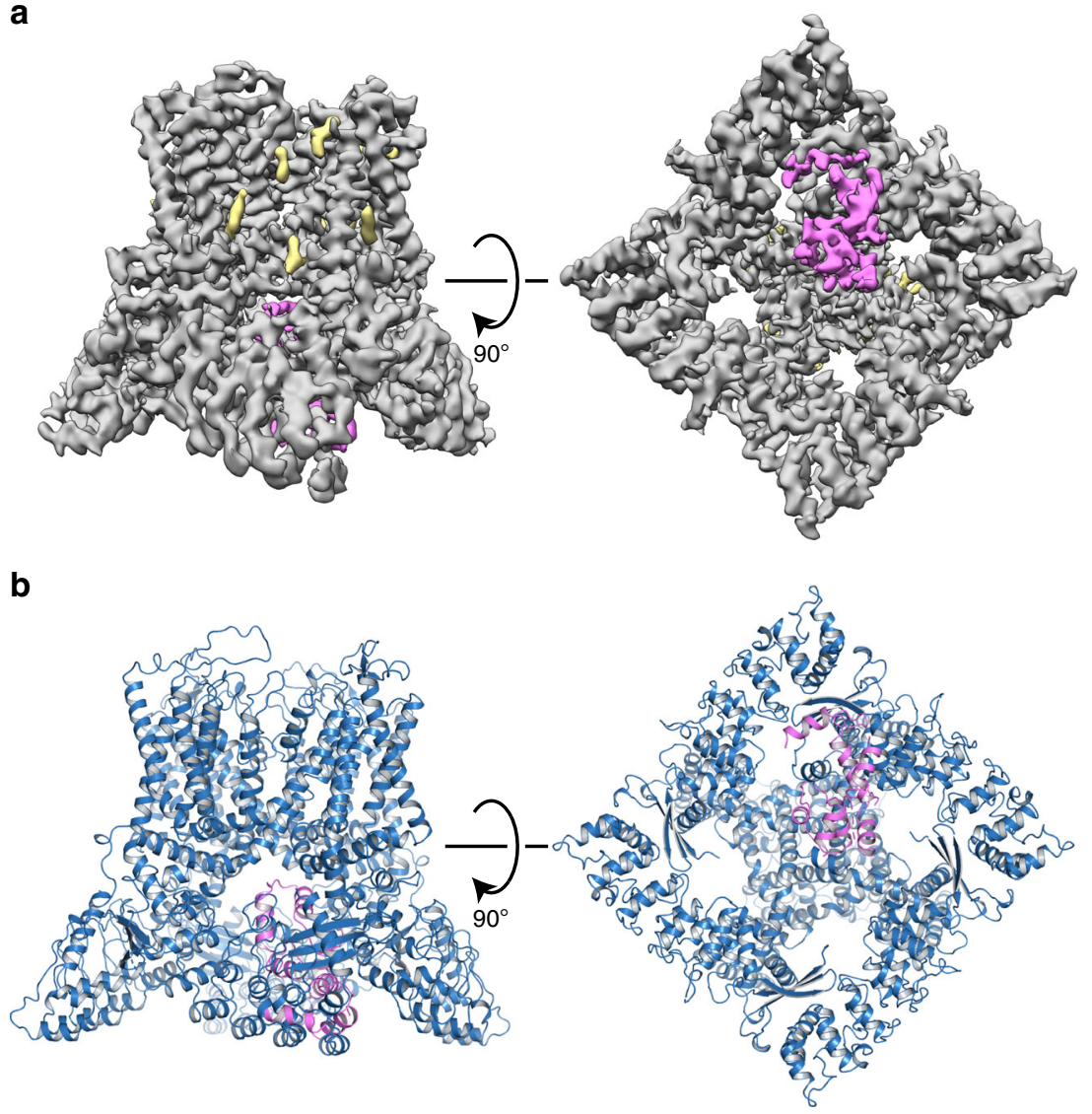

C
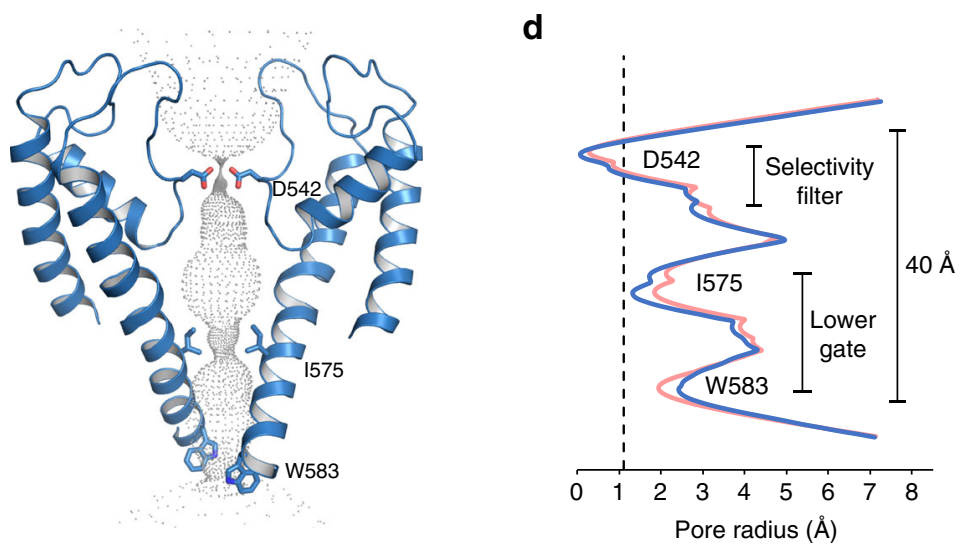

Fig. 5 CaM-bound structure of TRPV5. a Side and bottom view of the CaM-bound TRPV5 density map at $4.4 \AA$ resolution. Density for TRPV5 is shown in gray, annular lipids shown in khaki, and CaM shown in hot pink. b Cartoon representation of the CaM-bound TRPV5 model in a side and bottom view. TRPV5 is shown in blue and CaM is shown in hot pink. $\mathbf{c}$ Pore diagram of CaM-bound TRPV5. Constriction residues are labeled and shown as sticks. $\mathbf{d}$ Plot of pore radii of CaM-bound TRPV5 as a function of distance through the pore is shown in blue. For reference, the lipid-bound TRPV5 pore graph is shown in pink. The dotted line indicates the radius of a calcium ion

conformational changes in the pore, which allows for the flow of $\mathrm{Ca}^{2+}$ ions (Figs. 3 and 7).

The CaM-bound structure has provided the first structural insight into how TRPV5 ion flow is inhibited by CaM. It is clear that the binding of a single CaM molecule completely obstructs the intracellular side of the pore, effectively blocking ion permeation (Fig. 7). This study also showed that while CaM binds to the predicted areas in the C-terminus of TRPV5, it also directly interacts at an additional C-terminal-binding site (Fig. 6). Taken together, the lipid-bound and CaM-bound TRPV5 structures provide additional evidence for the static nature of some TRPV subfamily channels ${ }^{15,25}$. The minimal movement (RMSD $\sim 0.6 \AA$ ) between the two structures implies that large conformational changes in the upper and lower gates of TRPV5 may not be necessary for effective endogenous inhibition.

It has previously been shown that the $\mathrm{N}$-lobe and C-lobe of CaM have different affinities for calcium ions, with the C-lobe having a six-fold higher affinity for calcium ions than the $\mathrm{N}-$ lobe $^{27}$. Other groups have proposed that this allows the CaM C-lobe to be constantly bound to the distal C-terminus of TRPV5 at normal cellular calcium levels, keeping a constant supply of $\mathrm{CaM}$ near the pore $\mathrm{e}^{23,28}$. In this model, the $\mathrm{N}$-lobe only binds to the C-terminus of TRPV5 when calcium is high and the pore needs to be closed, and the second CaM binding event may 
a
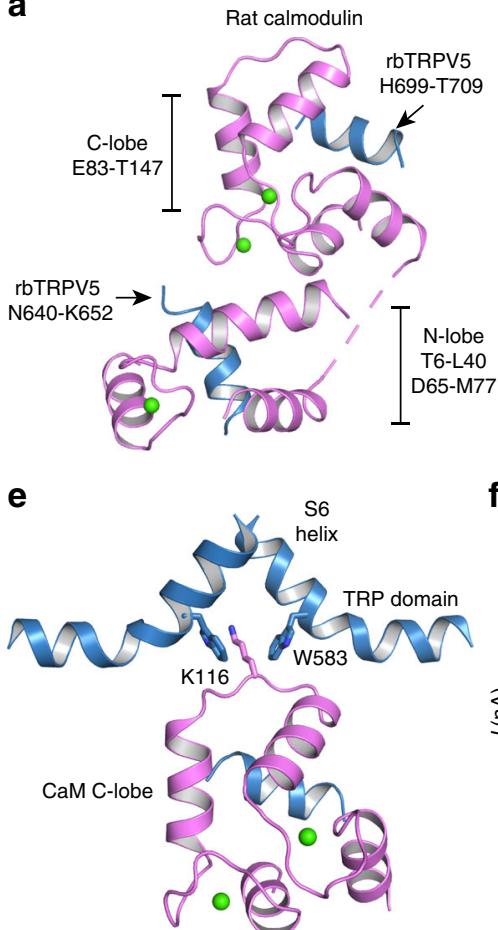

b

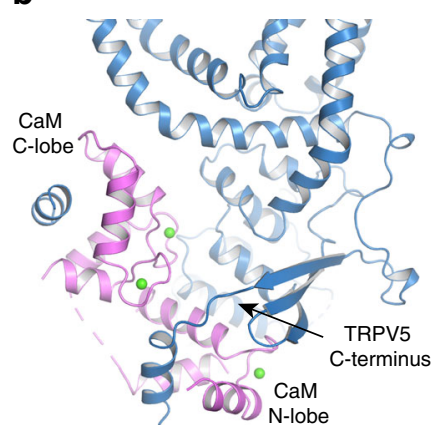

f

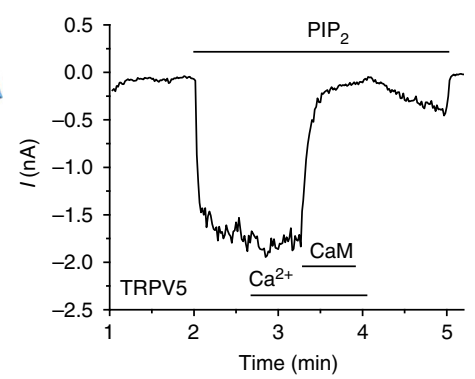

c

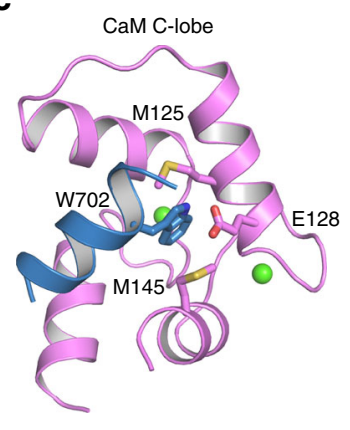

g

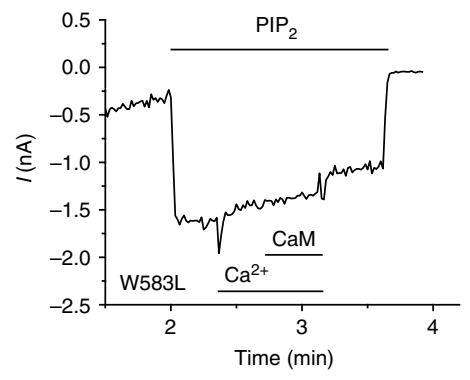

d

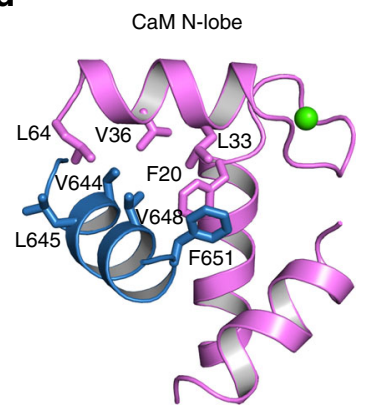

h

Fig. 6 Mechanism of TRPV5 inactivation by CaM. a Structure of rat CaM bound to TRPV5 C-terminus fragments. Both the N-lobe and C-lobe were visualized along with N640-K652 and H699-T709 of TRPV5. CaM is shown in hot pink, and TRPV5 is shown in blue. Calcium ions bound to the N-lobes and C-lobes of CaM are shown as green spheres. b CaM interaction with chain A of TRPV5. c CaM C-lobe interaction with W702 on the TRPV5 C-terminus. d CaM N-lobe makes multiple hydrophobic interactions along N640-K652. e The base of the TRPV5 pore is shown with K116 of CaM bound at W583, causing a steric blockage of calcium flow. $\mathbf{f}$ The excised inside out patch clamp experiments show that wild type TRPV 5 is inhibited upon addition of $\mathrm{Ca}^{2+}$ activated CaM. Representative current trace is at $-100 \mathrm{mV}$; the applications of $25 \mu \mathrm{M} \mathrm{diC} 8 \mathrm{PI}(4,5) \mathrm{P}_{2}, 0.2 \mu \mathrm{M} \mathrm{CaM}$ and $3 \mu \mathrm{M}$ free Ca ${ }^{2+}$ are indicated

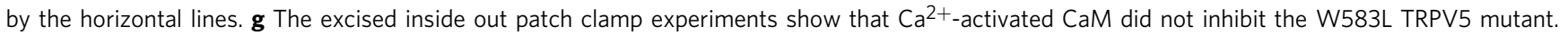
Representative current trace is at $-100 \mathrm{mV}$; the applications of $25 \mu \mathrm{M} \mathrm{diC} 8 \mathrm{PI}(4,5) \mathrm{P}_{2}, 0.2 \mu \mathrm{M} \mathrm{CaM}$ and $3 \mu \mathrm{M}$ free Ca ${ }^{2+}$ are indicated by the horizontal lines. h Summary of the data, current amplitudes after CaM were divided by current values before CaM applications, $P=0.011$ (two-sample $t$-test). The sample size $(n)$ indicates the number of individual patches tested, each obtained from a different oocyte. Error bars represent \pm SEM

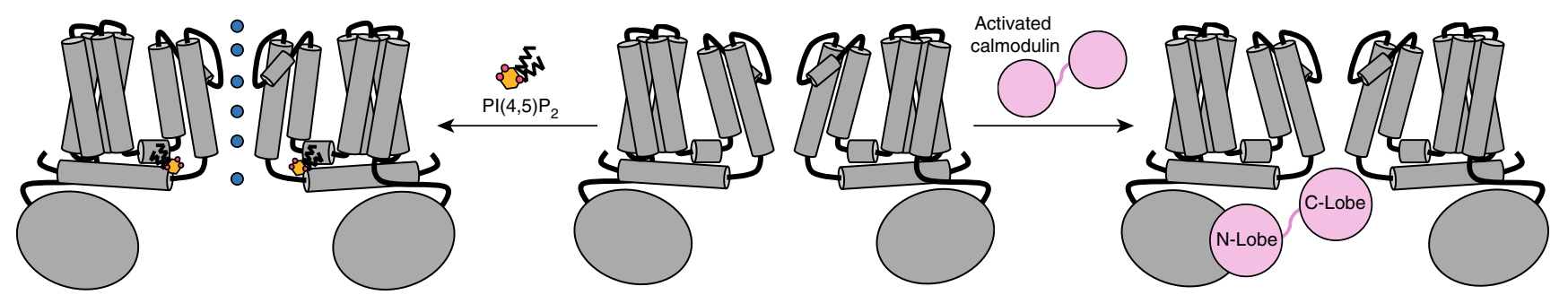

Fig. 7 TRPV5 activation by $\mathrm{PI}(4,5) \mathrm{P}_{2}$ and inactivation by CaM. A schematic representation of the proposed binding of both $\mathrm{PI}(4,5) \mathrm{P}_{2}$ and CaM to TRPV5, inducing either channel activation or inactivation. A dimer of TRPV 5 is shown as a gray diagram, $\mathrm{PI}(4,5) \mathrm{P}_{2}$ is shown in orange, CaM is shown in pink and the blue circles indicate the flow of calcium ions

stabilize the position of $\mathrm{CaM}$ at the base of the pore and lead to the occlusion of the channel23,28. The CaM N-lobe-binding site at TRPV5 residues Asn640-Lys652 could compliment the previous model, by potentially "reeling in" CaM when calcium levels are high and bringing the CaM C-lobe into close proximity with the base of the pore.

Overall, our structural studies have provided molecular insights into the endogenous modulation of TRPV5 that could guide therapeutics design for a variety of calcium dependent kidney diseases.

\section{Methods}

Protein expression and purification. Full-length rabbit TRPV5 was expressed with a 1D4 affinity tag in Saccharomyces cerevisiae ${ }^{29,30}$. The membranes were lysed and harvested using a M-110Y microfluidizer and ultracentrifugation. Lysed membranes containing TRPV5 were solubilized in Buffer A $(150 \mathrm{mM} \mathrm{NaCl}, 2 \mathrm{mM}$ TCEP, $1 \mathrm{mM}$ PMSF, $20 \mathrm{mM}$ HEPES $\mathrm{pH} 8,10 \%$ glycerol, and $0.87 \mathrm{mM}$ LMNG). Non-soluble material was removed via ultracentrifugation. Detergent solubilized TRPV5 was then purified using CnBr-activated Sepharose $4 \mathrm{~B}$ beads conjugated to 1D4 specific antibodies. The beads were washed with Buffer B $(150 \mathrm{mM} \mathrm{NaCl}$, $2 \mathrm{mM}$ TCEP, $20 \mathrm{mM}$ HEPES $\mathrm{pH}$ 8, and $0.064 \mathrm{mM}$ DMNG) and TRPV5 was eluted using Buffer B with the addition of $3 \mathrm{mg} / \mathrm{mL} 1 \mathrm{D} 4$ peptide.

The sample involved in the reconstruction of lipid-bound TRPV5 in detergent was then subjected to size-exclusion chromatography (Superose 6, GE Healthcare) in Buffer B. This sample was concentrated to $\sim 2.5 \mathrm{mg} / \mathrm{mL}$ and incubated with soluble $\mathrm{diC}_{8} \mathrm{PI}(4,5) \mathrm{P}_{2}$ (dioctanoyl phosphatidylinositol 4,5-bisphosphate) at a final concentration of $200 \mu \mathrm{M}$ for 30 min prior to vitrification.

For the sample that was reconstituted into nanodiscs, after elution from the Sepharose beads TRPV5 was incubated with MSP2N2 and soy polar lipid extract (Avanti Polar Lipids, Inc.) in a molar ratio of 1:1:200 (TRPV5:MSP2N2:Soy Polar Lipids) for $30 \mathrm{~min}$. To produce purified MSP2N2 for the reconstitution, MSP2N2 
(Addgene) was transformed into BL21 (DE3) cells, then grown on LB plates containing $30 \mu \mathrm{g} / \mathrm{mL}$ kanamycin. Cells expressing MSP2N2 were then grown in TB supplemented with $0.2 \%$ glucose and $30 \mu \mathrm{g} / \mathrm{mL}$ kanamycin at $37^{\circ} \mathrm{C}$ with shaking. When the culture reached an $\mathrm{OD}_{600}$ of $\sim 0.5$ the temperature was reduced to $28^{\circ} \mathrm{C}$. The culture was induced with $1 \mathrm{mM}$ IPTG at an $\mathrm{OD}_{600}$ of $\sim 1.0$. After $3 \mathrm{~h}$ the culture was harvested by centrifugation and the cell pellet was resuspended in a buffer containing $100 \mathrm{mM} \mathrm{NaCl}, 20 \mathrm{mM}$ Tris- $\mathrm{HCl}, \mathrm{pH}$ 7.4, $1 \mathrm{mM}$ PMSF and a complete EDTA-free protease inhibitor cocktail tablet (Roche). Cells were lysed via homogenization and centrifuged at $30,000 \times g$ for $30 \mathrm{~min}$. The lysate supernatant was bound to Ni-NTA equilibrated with Buffer C $(100 \mathrm{mM} \mathrm{NaCl}, 20 \mathrm{mM}$ Tris- $\mathrm{HCl}, \mathrm{pH}$ 7.4). The bound resin was washed with four bed volumes of Buffer $\mathrm{C}$ containing $1 \%$ Triton X-100 followed by four bed volumes of Buffer C supplemented with $50 \mathrm{mM}$ sodium cholate and four bed volumes of Buffer $\mathrm{C}$ containing $20 \mathrm{mM}$ imidazole. The MSP2N2 was eluted from the Ni-NTA resin with Buffer C supplemented with $300 \mathrm{mM}$ imidazole. The elution was passed through a desalting column equilibrated with $50 \mathrm{mM}$ Tris- $\mathrm{HCl}, \mathrm{pH}$ 7.5, $100 \mathrm{mM}$ $\mathrm{NaCl}$, and $0.5 \mathrm{mM}$ EDTA. Desalted MSP2N2 was utilized for reconstitution ${ }^{31}$. Soy polar lipids were dried under nitrogen flow for $3 \mathrm{~h}$ prior to reconstitution and dried lipids were resuspended with a 1:200 molar ratio of lipids to DMNG before being added to the protein mixture. Bio-Beads (Bio-Rad, Bio-Beads SM-2 Absorbent Media) were added to the TRPV5, MSP, lipid mixture for $1 \mathrm{~h}$. Fresh Bio-Beads were then added and allowed to incubate overnight. The reconstituted nanodiscs were further purified using size-exclusion chromatography (Superose 6, GE Healthcare) in buffer containing $150 \mathrm{mM} \mathrm{NaCl}, 2 \mathrm{mM}$ TCEP, and $20 \mathrm{mM}$ HEPES $\mathrm{pH} 8$. This sample was concentrated to $\sim 2.8 \mathrm{mg} / \mathrm{mL}$ and incubated with soluble $\mathrm{diC}_{8} \mathrm{PI}(4,5) \mathrm{P}_{2}$ at a final concentration of $400 \mu \mathrm{M}$ for $30 \mathrm{~min}$ prior to vitrification.

Rat CaM in the pET 28b vector was expressed in Rosetta 2(DE3) Escherichia coli. Cells were grown in Terrefic Brothe supplemented with $30 \mu \mathrm{g} / \mathrm{mL}$ kanamycin and $35 \mu \mathrm{g} / \mathrm{mL}$ chlorempinacole at $37^{\circ} \mathrm{C}$. The cells were resuspended in buffer containing $20 \mathrm{mM}$ Tris, $150 \mathrm{mM} \mathrm{NaCl}, 1 \mathrm{mM}$ EDTA, $\mathrm{pH}$ 7.5, $1 \mathrm{mM}$ PMSF, and a complete EDTA-free protease inhibitor cocktail tablet (Roche) then sonicated for $1 \mathrm{~h}$. The lysate was passed through low substitution phenyl sepharose fast flow column (GE Healthcare) equilibrated with $20 \mathrm{mM}$ Tris, $1 \mathrm{mM}$ EGTA, pH 7.5 buffer. The flow through was then supplemented with $20 \mathrm{mM} \mathrm{CaCl}_{2}$ and run through a low substitution phenyl sepharose fast flow column equilibrated with $20 \mathrm{mM}$ Tris, $500 \mathrm{mM} \mathrm{NaCl}, 1 \mathrm{mM} \mathrm{CaCl}$, pH 7.5. The column was washed with $20 \mathrm{mM}$ Tris, $1 \mathrm{mM} \mathrm{CaCl}_{2}, \mathrm{pH} 7.5$, and the CaM was eluted with $20 \mathrm{mM}$ Tris, $1 \mathrm{mM}$ EGTA, pH 7.5. CaM was further purified using an AKTA purifier (GE Healthcare) with the following buffer: $10 \mathrm{mM}$ Tris, $50 \mathrm{mM} \mathrm{NaCl}, 10 \mathrm{mM} \mathrm{CaCl}_{2}, \mathrm{pH} 7.5^{32}$. Purified TRPV5 was incubated for $1 \mathrm{~h}$ with $10 \mathrm{mM} \mathrm{CaCl}_{2}$ and the purified rat CaM at a molar ratio of 1:20 (TRPV5 tetramer:CaM) after elution from the Sepharose beads. The TRPV5-CaM mixture was then further purified using size-exclusion chromatography (Superose 6, GE Healthcare) in Buffer B. No chelating agents were added to Buffer B; thus, trace amounts of calcium are present in the final sample. The peak containing CaM-bound TRPV5 was concentrated to $\sim 3 \mathrm{mg} / \mathrm{mL}$.

Cryo-EM data acquisition. For all samples, fluorinated Fos-Choline-8 was added to the concentrated protein to a final concentration of $3 \mathrm{mM}$ immediately prior to vitrification. Samples were double blotted on 200 mesh Quantifoil 1.2/1.3 grids (Quantifoil Micro Tools) with $3.5 \mu \mathrm{L}$ per blot and plunge frozen in liquid ethane using a Vitrobot (Thermo Fisher Scientific). Grids containing TRPV5 and $\mathrm{diC}_{8} \mathrm{PI}$ $(4,5) \mathrm{P}_{2}$ in detergent, as well as grids with CaM-bound TRPV5 were imaged with a $300 \mathrm{kV}$ Titan Krios microscope equipped with a Gatan K2 Summit direct detector camera. Super resolution movies (50 frames) were captured for $10 \mathrm{~s}$ each with one frame collected every $0.2 \mathrm{~s}$. The resultant pixel size and dose rate were $0.55 \AA /$ pix and $\sim 8$ electrons/pix/s, respectively. Images were collected in a defocus range between 1.0 and $2.5 \mu \mathrm{m}$ under focus in an automated fashion using Leginon software $^{33}$

Grids containing TRPV5 and $\mathrm{diC}_{8} \mathrm{PI}(4,5) \mathrm{P}_{2}$ in nanodiscs were also imaged with a $300 \mathrm{kV}$ Titan Krios microscope equipped with a Gatan K2 Summit direct detector camera. Super resolution movies (40 frames) were captured with an $8 \mathrm{~s}$ exposure time in super resolution mode resulting in a pixel size and dose rate of $0.532 \AA / p i x$ and $\sim 6$ electrons/pix/sec, respectively. Images were collected in a defocus range between 1.25 and $2.5 \mu \mathrm{m}$ under focus.

Image processing. For the dataset of lipid-bound TRPV5 in detergent, MotionCor2 ${ }^{34}$ was used to correct for beam induced motion and to bin the images to a final pixel size of $1.1 \AA$, producing both summed and dose weighted micrographs. CTF estimation of the summed micrographs was preformed using $\mathrm{Gctf}^{35}$. All other image processing was performed using RELION on the dose weighted micrographs unless otherwise mentioned ${ }^{18,19}$. Approximately 2500 particles were picked manually from 3889 micrographs and sorted into $2 \mathrm{D}$ classes. The best classes were used as templates for autopicking. After autopicking, 2D classification was used to remove false positives and suboptimal particles. The remaining $\sim 241,000$ particles were reconstructed into a single electron density map with C4 symmetry using the $3 \mathrm{D}$ auto-refine option in RELION followed by 3D classification into eight classes without assigning angles to the particles. The initial model used for 3D refinement and classification was produced by applying a low-pass filter of $60 \AA$ to the previously published TRPV5 cryo-EM structure ${ }^{4}$. The best class by manual inspection underwent multiple rounds of 3D refinement followed by 3D classification until the best $\sim 26,000$ particles were able to be reconstructed into a $4.4 \AA$ map. The mask used for this reconstruction was created from the original 3D auto-refinement of $\sim 241,000$ particles adjusted to a threshold of 0.005 , low-pass filtered to $15 \AA$ and a soft edge of five pixels was applied. A separate dataset collected under the same conditions from grids prepared at the same time as the sample above produced 3062 additional micrographs. The same methods as above were implemented and resulted in a map containing $\sim 19,000$ particles that was reconstructed to $4.2 \AA$. These particles were then combined using the JoinStar command in Relion to produce a particle set of $\sim 45,000$ particles that were refined to $4.1 \AA$ in RELION (Supplementary Figure 2). A B-factor of -174 was then applied to the unsharpened map using Bfactor software and the final resolution of $3.9 \AA$ was determined using rmeasure software ${ }^{36,37}$. Local resolutions were estimated using the RESMAP software ${ }^{38}$

For the dataset of PI(4,5) $\mathrm{P}_{2}$-bound TRPV5 in nanodiscs, MotionCor2 ${ }^{34}$ was used to correct for beam-induced motion and to bin the images to a final pixel size of $1.1 \AA$, producing both summed and dose-weighted micrographs. CTF estimation of the summed micrographs was preformed using Gctf ${ }^{35}$. All other image processing was performed using RELION on the dose-weighted micrographs unless otherwise mentioned ${ }^{18,19}$. To create templates for autopicking, 2112 particles were manually picked from 1575 micrographs and sorted into 21 classes. The best classes were used to autopick 493,047 particles which were then subjected to $2 \mathrm{D}$ classification into 200 classes to remove false positives and suboptimal particles. The best classes contained 121,980 particles, which were refined to $4.4 \AA$ using 3D AutoRefine with C4 symmetry in the absence of a mask using the same initial model as in the lipid-bound structure. A mask was created of this structure by adjusting the threshold to 0.005 , lowpass filtering to $15 \AA$ and applying a soft edge of five pixels. The $4.4 \AA$ structure was postprocessed using the above mask to produce the Initial 3D Reconstruction at $4.3 \AA$ (Supplementary Figure 4). A cylinder with a radius of $40 \AA$ and height of $15 \AA$ was created in Chimera, centered on the pore at the height of the TRP helices. This cylinder was used to make a mask at a threshold of 0.5 , an extended edge of two pixels, and a soft edge of five pixels. This mask was then used for particle subtraction, where everything outside of this mask was subtracted, as previously described ${ }^{39}$. The subtracted particles were then sorted by $3 \mathrm{D}$ classification into five classes without assigning angles, yielding three high-resolution classes. Two of these classes had density for PI $(4,5) \mathrm{P}_{2}$ (total of $\sim 72,000$ particles) and one had no $\mathrm{PI}(4,5) \mathrm{P}_{2}$ density $(\sim 30,000$ particles). We swapped both sets of subtracted particles back to the unsubtracted particles and refined both sets using the mask previously used to refine the $\sim 121,000$ particle map. The $\sim 30,000$ particles for the class without $\mathrm{PI}(4,5) \mathrm{P}_{2}$ were refined to $4.5 \AA$ in RELION. Postprocessing was run on this data in RELION using the same mask as previously with an applied $b$ factor of -200 , leading to a final resolution of $4.4 \AA$ The $\sim 72,000$ particles were then sorted again by $3 \mathrm{D}$ classification into three classes without assigning angles. The best of these classes consisted of $~ 25,500$ particles and was refined to $4.1 \AA$ in RELION. Postprocessing was run on this data in RELION using the same mask as previously with an applied $b$ factor of -150 , leading to a final resolution of $4.0 \AA$ (Supplementary Figure 4). Local resolutions were estimated using RESMAP ${ }^{38}$.

For the dataset of CaM-bound TRPV5 in detergent, MotionCorr $2^{34}$ was used without gain correction and without binning to produce both summed and dose weighted micrographs with a final pixel size of $1.1 \AA$. CTF estimation of the summed micrographs was preformed using $\mathrm{Gct}^{35}$. All other image processing was performed using RELION on the dose weighted micrographs unless otherwise mentioned ${ }^{18,19}$. To create templates for autopicking, 3,125 particles were manually picked from 2118 micrographs and sorted into 30 classes. The best classes were used to autopick 391,648 particles which were then subjected to 2D classification into 100 classes to remove false positives and suboptimal particles. The best classes contained 153,776 particles, which were refined to $5.4 \AA$ using 3D AutoRefine with $\mathrm{C} 1$ symmetry in the absence of a mask using the same initial model as in the lipidbound structure. At this point, there was clearly density present in the region between the ARDs of the TRPV5 monomers, but it appeared to be asymmetrical. To make sure that information for all the asymmetrical features was aligned in the same orientation, we decided to use symmetry expansion. The $\sim 154,000$ particles from the initial $\mathrm{C} 1$ reconstruction were refined in $\mathrm{C} 4$ to generate symmetry operators. RELION symmetry expansion in $\mathrm{C} 4$ was used to expand the particle stack and ensure that all the information for CaM was present in all four possible orientations. A sphere of radius $25 \AA$ was created in Chimera and placed at the base of the pore so that the entire region with density for $\mathrm{CaM}$ was in the sphere. A mask was made of this sphere in RELION, at a threshold of 0.1 , extended by five pixels, and with a soft edge of five pixels. This spherical mask was used for particle subtraction, as described above, and then the subtracted particles were sorted into five classes with $3 \mathrm{D}$ classification. The resulting classes captured CaM in the four possible orientations, with approximately equal numbers of particles, as well as a class without CaM bound. The class with the best resolution was chosen, consisting of $\sim 129,000$ particles, and we reverted to the unsubtracted particles and refined these particles. There was still some heterogeneity in the CaM, so we further classified the particles into three classes, resulting in a class with $\sim 47,500$ particles that refined to $4.7 \AA$ in RELION. After postprocessing in RELION with an applied $b$ factor of -200 , this data reached a resolution of $4.4 \AA$ (Supplementary Figure 11). Local resolutions were estimated using RESMAP ${ }^{38}$. 
Model building. The previously published model of TRPV5 bound to econazole $(\mathrm{PDB} 6 \mathrm{~B} 5 \mathrm{~V})^{4}$ was used as an initial model and docked to the cryo-EM map of lipid-bound TRPV5. The model was manually adjusted in $\operatorname{Coot}^{40}$ and refined with imposed four-fold NCS using phenix.real_space_refinement ${ }^{41}$. The lipid-bound TRPV5 model was docked into the PI $(4,5) \mathrm{P}_{2}$-bound TRPV5 map and manually adjusted and refined as before. The lipid-bound TRPV5 model was docked into the CaM-bound TRPV5 map. To model CaM into the extra density, the N-lobe (residues 6-77) and C-lobe (residues 83-147) of a calcium-bound CaM structure (PDB 1CLL) were separated and independently fit in Chimera to the two lobes of density visible at the base of the pore. A portion of the N-lobe was visible only as broken density, and so was removed from the model. The TRPV5 C-terminal peptide from state 01 of PDB 5 OEO was fit into the remaining density near the C-lobe in Chimera. An extension of the C-terminus of TRPV5 chain A was modeled into helical density in the peptide-binding pocket of the CaM N-Lobe. This assembly was manually adjusted in Coot and refined without NCS using phenix.real_space_refinement.

The final models were randomized in PHENIX ${ }^{41}$ by $0.5 \AA$ and refined against one of the half maps. EMAN2 was used to generate FSC curves between this refined test model and each half map, as well as between the final model and the summed map for each model. The pore radii were generated using HOLE ${ }^{42}$. Figures were made in Pymol $^{43}$ and Chimera ${ }^{44}$.

\section{Xenopus oocyte electrophysiology. Oocyte sacs were extracted from female} Xenopus laevis frogs (Xenopus Express) and digested with $0.2 \mathrm{mg} / \mathrm{ml}$ collagenase (Sigma) in OR2 solution $(82.5 \mathrm{mM} \mathrm{NaCl}, 2 \mathrm{mM} \mathrm{KCl}, 1 \mathrm{mM} \mathrm{MgCl} 2$, and $5 \mathrm{mM}$ HEPES, pH 7.4) overnight at $18^{\circ} \mathrm{C}$. Oocytes were maintained in OR2 solution plus $1.8 \mathrm{mM} \mathrm{CaCl}_{2}$ and $1 \%$ penicillin/streptomycin (Mediatech) at $18^{\circ} \mathrm{C} . \mathrm{cRNA}$ was generated using the mMessage mMachine kit (Thermo Fisher); point mutations were introduced using the Quikchange mutagenesis kit (Agilent Genomics). cRNA $(20 \mathrm{ng})$ was injected using a nanoliter injector system (World Precision Instruments). The W583L mutant of TRPV5 was kept in OR2 with $0.1 \mathrm{mM} \mathrm{CaCl}$, to avoid $\mathrm{Ca}^{2+}$ overload and cell damage. The experiments were performed $48-72 \mathrm{~h}$ after injection. Purified CaM from bovine testes was purchased from Sigma.

Two-electrode voltage clamp (TEVC) measurements were performed as described earlier ${ }^{14}$; briefly oocytes were initially placed in a solution containing $97 \mathrm{mM} \mathrm{NaCl}, 2 \mathrm{mM} \mathrm{KCl}, 1 \mathrm{mM} \mathrm{MgCl}_{2}$, and $5 \mathrm{mM}$ HEPES, pH 7.4, where TRPV5 or TRPV6 currents are largely blocked by $\mathrm{Mg}^{2+}$ and trace amounts of $\mathrm{Ca}^{2+}$ in the medium. Monovalent currents were initiated with changing the solution containing $96 \mathrm{mM} \mathrm{LiCl}, 1 \mathrm{mM}$ EGTA, and $5 \mathrm{mM}$ HEPES, $\mathrm{pH}$ 7.4. Currents were recorded with thin-wall inner filament-containing glass pipettes (World Precision Instruments) filled with $3 \mathrm{M} \mathrm{KCl}$ in $1 \%$ agarose. Currents were recorded with a GeneClamp 500B amplifier (Molecular Devices using a ramp protocol from -100 to $+100 \mathrm{mV}$ $(0.25 \mathrm{mV} / \mathrm{ms})$, immediately preceded by a 100 -ms step to $-100 \mathrm{mV}$, applied once every second; holding potential was $0 \mathrm{mV}$.

Excised inside-out patch clamp experiments were performed as described earlier ${ }^{13}$ using borosilicate glass pipettes (World Precision Instruments) of $0.8-1.7 \mathrm{M} \Omega$ resistance. The electrode pipette solution contained $96 \mathrm{mM} \mathrm{LiCl}, 1 \mathrm{mM}$ EGTA, and $5 \mathrm{mM}$ HEPES, pH 7.4. After establishing giagohm resistance seals on devitellinized Xenopus oocytes, the currents were measured using an Axopath 200B amplifier (Molecular Devices). We used a ramp protocol from -100 to $100 \mathrm{mV}$, performed once a second, immediately preceded by a $100-\mathrm{ms}$ step to $-100 \mathrm{mV}$. The perfusion solution contained $93 \mathrm{mM}$ potassium gluconate, $5 \mathrm{mM}$ HEDTA, $5 \mathrm{mM}$ HEPES, with the $\mathrm{pH}$ adjusted to 7.4. To obtain $3 \mu \mathrm{M}$ free $\mathrm{Ca}^{2+}, 2.5 \mathrm{mM} \mathrm{Ca}^{2+}$ gluconate was added. The bath was connected with the ground electrode through an agar bridge. Animal procedures were approved by the Institutional Animal Care and Use Committee of Rutgers New Jersey Medical School, and all animal procedures were performed in accordance with the approved ethical guidelines.

TRPV6 computational modeling. We generated an almost full-length atomistic model of TRPV6 by performing comparative homology modeling using the structure of the closed state of TRPV1 as a template (PDB:3J5P). We extracted the pairwise sequence alignment between TRPV1 and TRPV6 from the multiple sequence alignment containing about 3000 sequences ${ }^{45}$. We then generated 288 models using ROSETTA ${ }^{46}$ and discarded the 50 ones with the lowest score. We performed clustering on the remaining 248 structures and found two major clusters differing by the conformation of the S2-S3 segment. Due to the absence of significant structural constraints from the template, we modeled this region of TRPV6 $\mathrm{ab}$ initio.

To investigate the binding mode of $\mathrm{PI}(4,5) \mathrm{P}_{2}$ we considered a model ligand and performed a series of stochastic optimizations of its positions with respect to the TRPV6 structure using the program AUTODOCK ${ }^{47}$. The model ligand is a simplified version $\mathrm{PI}(4,5) \mathrm{P}_{2}$ that contains the entire head group, the glycerol moiety and part of the acyl chains (up to the third carbon atom). This choice allowed us to explore thoroughly the conformational space by ignoring the large number of degrees of freedom describing the configuration of the flexible acyl chain. Since not all the binding poses of the model ligand correspond to a geometrically viable conformation of $\mathrm{PI}(4,5) \mathrm{P}_{2}$, we post-processed the output of docking and selected only those binding poses in which the acyl chain is correctly positioned with respect to the lipid bilayer. The conformational search was restricted to a specific region of the channel encompassing the S4-S5 linker, the
$\mathrm{N}$-term sections of S1, S3, and S5, the C-term section and S2 and S4, the S2-S3 loop, the N-term section of the TRP box and the adjacent part of the cytoplasmic domain. The region was large enough to contain at least two adjacent subunits to allow for the identification of potential interactions at their interface. For each structure of TRPV6 (20 in total) 1000 docking experiments were performed using the Lamarckian genetic algorithm allowing for a maximum of 250,000 energy evaluations.

We performed molecular dynamics simulations of the PI(4,5) $\mathrm{P}_{2}$-TRPV6 and PI $(4,5) \mathrm{P}_{2}$-TRPV1 complexes and of the TRPV6 apo channel (with no bound PI $(4,5)$ $\mathrm{P}_{2}$ ). We used the naturally occurring long acyl chain version (arachydonyl-stearyl, AASt) $\mathrm{PI}(4,5) \mathrm{P}_{2}$ for these simulation. As initial configurations, we considered the structural models with the highest scores. The channels were embedded into a fully hydrated 1-palmitoyl-2-oleoyl-sn-glycero-3-phosphocholine membrane surrounded by a $150 \mathrm{mM} \mathrm{KCl}$ solution. Overall, the system contains more than 300,000 atoms. During the initial equilibration protocol, we gradually released each component of the (water and ions, lipid tails, lipid head groups, protein side chains and protein backbone) system from positional restraints. During the production run, trajectories were collected for $0.7 \mu \mathrm{s}$. Simulations were performed at constant temperature and pressure $(1 \mathrm{~atm}, 300 \mathrm{~K})$ using the Langevin piston approach using NAMD $2.10^{48}$. The CHARMM36 force field ${ }^{49}$ was used to describe the protein, the ions and the lipid molecules. For the vdW interactions, we used a cutoff of $11 \AA$ with a switching function between 8 and $11 \AA$. The long-range component of electrostatic interactions was calculated using the particle mesh Ewald approach ${ }^{50}$ using a cutoff for the short-range component of $11 \AA$. The equations of motion were integrated using the RESPA multiple time-step algorithm ${ }^{51}$, with a time step of $2 \mathrm{fs}$, and long-range interactions calculated every other step.

\section{Data availability}

The cryo-EM density maps and atomic coordinates of all structures presented in the text are deposited into the Electron Microscopy Data Bank and Protein Data Bank under the following access codes: Lipid-Bound TRPV5 in detergent (EMB-7965, PDB 6DMR); PI $(4,5) \mathrm{P}_{2}$-Bound TRPV5 in nanodiscs (EMB-7966, PDB 6DMU); CaM-Bound TRPV5 in detergent (EMB-7967, PDB 6DMW). All data is available from the corresponding author upon reasonable request.

Received: 7 June 2018 Accepted: 14 September 2018

Published online: 10 October 2018

\section{References}

1. Zhou, Y. \& Greka, A. Calcium-permeable ion channels in the kidney. Am. J. Physiol. Ren. Physiol. 310, F1157-F1167 (2016).

2. Na, T. \& Peng, J. B. TRPV5: a $\mathrm{Ca}(2+)$ channel for the fine-tuning of $\mathrm{Ca}(2+)$ reabsorption. Handb. Exp. Pharmacol. 222, 321-357 (2014).

3. van Goor, M. K. C., Hoenderop, J. G. J. \& van der Wijst, J. TRP channels in calcium homeostasis: from hormonal control to structure-function relationship of TRPV5 and TRPV6. Biochim. Biophys. Acta 1864, 883-893 (2017).

4. Hughes, T. E. T. et al. Structural basis of TRPV5 channel inhibition by econazole revealed by cryo-EM. Nat. Struct. Mol. Biol. 25, 53-60 (2018).

5. Lee, J., Cha, S. K., Sun, T. J. \& Huang, C. L. PIP2 activates TRPV5 and releases its inhibition by intracellular Mg2+. J. Gen. Physiol. 126, 439-451 (2005).

6. Rohacs, T., Lopes, C. M., Michailidis, I. \& Logothetis, D. E. PI(4,5)P2 regulates the activation and desensitization of TRPM8 channels through the TRP domain. Nat. Neurosci. 8, 626-634 (2005).

7. Thyagarajan, B., Lukacs, V. \& Rohacs, T. Hydrolysis of phosphatidylinositol 4,5-bisphosphate mediates calcium-induced inactivation of TRPV6 channels J. Biol. Chem. 283, 14980-14987 (2008).

8. Zakharian, E., Cao, C. \& Rohacs, T. Intracellular ATP supports TRPV6 activity via lipid kinases and the generation of $\operatorname{PtdIns}(4,5)$ P. FASEB J. 25 , 3915-3928 (2011)

9. Nilius, B. et al. The carboxyl terminus of the epithelial $\mathrm{Ca}(2+)$ channel $\mathrm{ECaCl}$ is involved in $\mathrm{Ca}(2+)$-dependent inactivation. Pflug. Arch. 445, 584-588 (2003).

10. de Groot, T. et al. Molecular mechanisms of calmodulin action on TRPV5 and modulation by parathyroid hormone. Mol. Cell. Biol. 31, 2845-2853 (2011).

11. Derler, I. et al. Dynamic but not constitutive association of calmodulin with rat TRPV6 channels enables fine tuning of $\mathrm{Ca} 2+$-dependent inactivation. J. Physiol. 577, 31-44 (2006).

12. Niemeyer, B. A., Bergs, C., Wissenbach, U., Flockerzi, V. \& Trost, C. Competitive regulation of CaT-like-mediated $\mathrm{Ca} 2+$ entry by protein kinase $\mathrm{C}$ and calmodulin. Proc. Natl Acad. Sci. USA 98, 3600-3605 (2001).

13. Cao, C., Zakharian, E., Borbiro, I. \& Rohacs, T. Interplay between calmodulin and phosphatidylinositol 4,5-bisphosphate in $\mathrm{Ca} 2+$-induced inactivation of transient receptor potential vanilloid 6 channels. J. Biol. Chem. 288, 5278-5290 (2013).

14. Velisetty, P. et al. A molecular determinant of phosphoinositide affinity in mammalian TRPV channels. Sci. Rep. 6, 27652 (2016). 
15. McGoldrick, L. L. et al. Opening of the human epithelial calcium channel TRPV6. Nature 553, 233-237 (2018).

16. Saotome, K., Singh, A. K., Yelshanskaya, M. V. \& Sobolevsky, A. I. Crystal structure of the epithelial calcium channel TRPV6. Nature 534, 506-511 (2016).

17. Singh, A. K., Saotome, K. \& Sobolevsky, A. I. Swapping of transmembrane domains in the epithelial calcium channel TRPV6. Sci. Rep. 7, 10669 (2017).

18. Scheres, S. H. RELION: implementation of a Bayesian approach to cryo-EM structure determination. J. Struct. Biol. 180, 519-530 (2012).

19. Scheres, S. H. Processing of structurally heterogeneous cryo-EM data in RELION. Methods Enzymol. 579, 125-157 (2016).

20. Rohacs, T. Phosphoinositide regulation of TRP channels. Handb. Exp. Pharmacol. 223, 1143-1176 (2014).

21. van der Wijst, J. et al. A gate hinge controls the epithelial calcium channel TRPV5. Sci. Rep. 7, 45489 (2017).

22. Nilius, B. et al. The carboxyl terminus of the epithelial $\mathrm{Ca}(2+)$ channel $\mathrm{ECaCl}$ is involved in $\mathrm{Ca}(2+)$-dependent inactivation. Pflug. Arch. 445, 584-588 (2003).

23. Bokhovchuk, F. M. et al. The structural basis of calcium-dependent inactivation of the transient receptor potential vanilloid 5 channel. Biochemistry 57, 2623-2635 (2018).

24. Holakovska, B., Grycova, L. \& Bily, J. Characterization of calmodulin binding domains in TRPV2 and TRPV5 C-tails. Amino Acids 40, 741-748 (2011).

25. Gao, Y., Cao, E., Julius, D. \& Cheng, Y. TRPV1 structures in nanodiscs reveal mechanisms of ligand and lipid action. Nature 534, 347-351 (2016).

26. Poblete, $\mathrm{H}$. et al. Molecular determinants of phosphatidylinositol 4,5bisphosphate (PI $(4,5) \mathrm{P} 2)$ binding to transient receptor potential V1 (TRPV1) channels. J. Biol. Chem. 290, 2086-2098 (2015).

27. Linse, S., Helmersson, A. \& Forsen, S. Calcium binding to calmodulin and its globular domains. J. Biol. Chem. 266, 8050-8054 (1991).

28. Bate, N. et al. A novel mechanism for calmodulin-dependent inactivation of transient receptor potential vanilloid 6. Biochemistry 57, 2611-2622 (2018).

29. Moiseenkova, V. Y., Hellmich, H. L. \& Christensen, B. N. Overexpression and purification of the vanilloid receptor in yeast (Saccharomyces cerevisiae). Biochem. Biophys. Res. Commun. 310, 196-201 (2003).

30. Moiseenkova-Bell, V. Y., Stanciu, L. A., Serysheva, I. I., Tobe, B. J. \& Wensel, T. G. Structure of TRPV1 channel revealed by electron cryomicroscopy. Proc. Natl Acad. Sci. USA 105, 7451-7455 (2008).

31. Basak, S., Schmandt, N., Gicheru, Y., Chakrapani, S. Crystal structure and dynamics of a lipid-induced potential desensitized-state of a pentameric ligand-gated channel. eLife 6, e23886 (2017).

32. Zhang, M. et al. Selective phosphorylation modulates the PIP2 sensitivity of the CaM-SK channel complex. Nat. Chem. Biol. 10, 753-759 (2014).

33. Potter, C. S. et al. Leginon: a system for fully automated acquisition of 1000 electron micrographs a day. Ultramicroscopy 77, 153-161 (1999).

34. Zheng, S. Q. et al. MotionCor2: anisotropic correction of beam-induced motion for improved cryo-electron microscopy. Nat. Methods 14, 331-332 (2017).

35. Zhang, K. Gctf: real-time CTF determination and correction. J. Struct. Biol. 193, 1-12 (2016).

36. Grigorieff, N. Resolution measurement in structures derived from single particles. Acta Crystallogr. D Biol. Crystallogr. 56, 1270-1277 (2000).

37. Sousa, D. \& Grigorieff, N. Ab initio resolution measurement for single particle structures. J. Struct. Biol. 157, 201-210 (2007).

38. Kucukelbir, A., Sigworth, F. J. \& Tagare, H. D. Quantifying the local resolution of cryo-EM density maps. Nat. Methods 11, 63-65 (2014).

39. Bai, X. C., Rajendra, E., Yang, G., Shi, Y., Scheres, S. H. Sampling the conformational space of the catalytic subunit of human gamma-secretase. eLife 4, el1182 (2015).

40. Emsley, P. \& Cowtan, K. Coot: model-building tools for molecular graphics. Acta Crystallogr. D Biol. Crystallogr. 60, 2126-2132 (2004).

41. Adams, P. D. et al. PHENIX: a comprehensive Python-based system for macromolecular structure solution. Acta Crystallogr. D Biol. Crystallogr. 66 , 213-221 (2010).

42. Smart, O. S., Neduvelil, J. G., Wang, X., Wallace, B. A. \& Sansom, M. S. HOLE: a program for the analysis of the pore dimensions of ion channel structural models. J. Mol. Graph. 14, 354-360 (1996). 376.

43. Alexander, N., Woetzel, N. \& Meiler, J. bcl::Cluster: a method for clustering biological molecules coupled with visualization in the Pymol molecular graphics system. IEEE Int Conf. Comput. Adv. Bio Med Sci. 2011, 13-18 (2011).

44. Pettersen, E. F. et al. UCSF Chimera-a visualization system for exploratory research and analysis. J. Comput. Chem. 25, 1605-1612 (2004).

45. Palovcak, E., Delemotte, L., Klein, M. L. \& Carnevale, V. Comparative sequence analysis suggests a conserved gating mechanism for TRP channels. J. Gen. Physiol. 146, 37-50 (2015).

46. Kaufmann, K. W., Lemmon, G. H., Deluca, S. L., Sheehan, J. H. \& Meiler, J. Practically useful: what the Rosetta protein modeling suite can do for you. Biochemistry 49, 2987-2998 (2010).
47. Morris, G. M. et al. AutoDock4 and AutoDockTools4: automated docking with selective receptor flexibility. J. Comput. Chem. 30, 2785-2791 (2009).

48. Phillips, J. C. et al. Scalable molecular dynamics with NAMD. J. Comput. Chem. 26, 1781-1802 (2005).

49. Mackerell, A. D.Jr., Feig, M. \& Brooks, C.L.III. Extending the treatment of backbone energetics in protein force fields: limitations of gas-phase quantum mechanics in reproducing protein conformational distributions in molecular dynamics simulations. J. Comput. Chem. 25, 1400-1415 (2004).

50. Darden, T., York, D. \& Pedersen, L. Particle mesh Ewald -an N.Log(N) method for Ewald sums in large systems. J. Chem. Phys. 98, 10089-10092 (1993).

51. Tuckerman, M., Berne, B. J. \& Martyna, G. J. Reversible multiple time scale molecular-dynamics. J. Chem. Phys. 97, 1990-2001 (1992).

\section{Acknowledgements}

We thank Dr. Ji-fang Zhang and Dr. AjaySingh Tanwar at Thomas Jefferson University for the gift of purified rat calmodulin. We thank Denice Major for assistance with hybridoma and cell culture at the Department of Ophthalmology and Visual Sciences (supported by the National Institutes of Health Core Grant P30EY11373). We thank Yvonne Gicheru and Dr. Sudha Chakrapani at Case Western Reserve University for training and assistance with MSP2N2 expression and purification. We acknowledge the use of instruments at the Electron Imaging Center for NanoMachines supported by NIH (1S10RR23057 and 1S10OD018111), NSF (DBI-1338135 and DMR-1548924), and CNSI at UCLA. We also acknowledge microscopist Carol Bator and the use of instrument at the Penn State Cryo Electron Microscopy Facility (University Park, PA). This research was, in part, supported by the National Cancer Institute's National Cryo-EM Facility at the Frederick National Laboratory for Cancer Research. We acknowledge the use of instruments at the Electron Microscopy Resource Lab at the University of Pennsylvania. We thank Dr. David Lodowski at Case Western Reserve University for help in the early stage of the project. This work was supported by grants from the National Institute of Health (R01GM103899 to V.Y.M.-B., R01GM093290 to T.R. and V.C., U24 GM116792 to Z.H.Z and V.Y.M.-B).

\section{Author contributions}

T.E.T.H. conducted protein purification, cryo-EM sample preparation and cryo-EM data collection; T.E.T.H. and R.A.P. performed all cryo-EM data analysis and interpretation; R.A.P. built and refined all atomic models; A.T.Y. performed all electrophysiology experiments and analyzed the resultant data; M.A.K. performed all TRPV6 modeling, docking, and molecular dynamic simulations; E.C.F. assisted T.E.T.H. and R.A.P. in data analysis; K.W.H. assisted T.E.T.H. in cryo-EM data collection; A.S. and S.M. trained and assisted T.E.T.H. in cryo-EM sample preparation and screening; Z.H.Z. supervised cryoEM data collection; V.C. supervised TRPV6 simulations and the subsequent data interpretation; T.R. supervised the electrophysiology experiments and assisted with data analysis; V.Y.M-B. designed and supervised the execution of all experiments in this manuscript; T.E.T.H., R.A.P., T.R. and V.Y.M.-B. wrote the final version of the manuscript; All authors contributed to and reviewed the final manuscript.

\section{Additional information}

Supplementary Information accompanies this paper at https://doi.org/10.1038/s41467018-06753-6.

Competing interests: The authors declare no competing interests.

Reprints and permission information is available online at http://npg.nature.com/ reprintsandpermissions/

Publisher's note: Springer Nature remains neutral with regard to jurisdictional claims in published maps and institutional affiliations.

Open Access This article is licensed under a Creative Commons Attribution 4.0 International License, which permits use, sharing, adaptation, distribution and reproduction in any medium or format, as long as you give appropriate credit to the original author(s) and the source, provide a link to the Creative Commons license, and indicate if changes were made. The images or other third party material in this article are included in the article's Creative Commons license, unless indicated otherwise in a credit line to the material. If material is not included in the article's Creative Commons license and your intended use is not permitted by statutory regulation or exceeds the permitted use, you will need to obtain permission directly from the copyright holder. To view a copy of this license, visit http://creativecommons.org/ licenses/by/4.0\%

(C) The Author(s) 2018 